\title{
Transcriptome analysis and functional identification of GmMYB46 in soybean seedlings under salt stress
}

\author{
Xun Liu ${ }^{1,2}$, Xinxia Yang ${ }^{3}$, Bin Zhang ${ }^{\text {Corresp. } 1}$ \\ ${ }^{1}$ College of Chemistry and Bioengineering, Hunan University of Science and Engineerireng, Yongzhou, China \\ 2 College of Life Sciences, Nanjing Agricultural University, Nanjing, China \\ 3 Department of Logistics, Hunan University of Science and Engineering, Yongzhou, China \\ Corresponding Author: Bin Zhang \\ Email address: zhangbin27104@huse.edu.cn
}

Salinit $y$ is one of the major abiotic stress that limits crop growth and productivity. We investigated the transcriptomes of salt-treated soybean seedlings versus a control using RNA-seq to better understand the molecular mechanisms of the soybean (Glycine max L.) response to salt stress. T ranscriptome analysis revealed 1,235 differentially expressed genes (DEGs) under salt stress. Several important pathways and key candidate genes were identified by KEGG enrichment. A total of 116 differentially expressed transcription factors (TFs) were identified, and 17 TFs were found to belong to MYB families. Phylogenetic analysis revealed that these TFs may be involved in salt stress adaptation. Further analysis revealed that GmMYB46 was up-regulated by salt and mannitol and was localized in the nucleus. The salt tolerance of transgenic Arabidopsis overexpressing GmMYB46 was significantly enhanced compared to wild-type (WT). GmMYB46 activates the expression of salt stress response genes (P5CS1, SOD, POD, NCED3) in Arabidopsis under salt stress, indicating that the GmMYB46 protein mediates the salt stress response through complex regulatory mechanisms. This study provides information with which to better understand the molecular mechanism of salt tolerance in soybeans and to genetically improve the crop. 
1 Transcriptome analysis and functional identification of GmMYB46 in

\section{2 soybean seedlings under salt stress}

3 Xun Liu ${ }^{1,2}$, Xinxia Yang ${ }^{3}$, Bin Zhang ${ }^{1 *}$

4 1. College of Chemistry and Bioengineering, Hunan University of Science and Engineering, Yongzhou 425199, China

5 2. College of Life Sciences, Nanjing Agricultural University, Nanjing 210095, China

6 3. Department of Logistics, Hunan University of Science and Engineering, Yongzhou 425199, China;

$7 \quad$ *Correspondence: zhangbin27104@huse.edu.cn

\section{ABSTRACT}

Salinity is one of the major abiotic stress that limits crop growth and productivity. We investigated the transcriptomes of salt-treated soybean seedlings versus a control using RNA-seq to better understand the molecular mechanisms of the soybean (Glycine max L.) response to salt stress. Transcriptome analysis revealed 1,235 differentially expressed genes (DEGs) under salt stress. Several important pathways and key candidate genes were identified by KEGG enrichment. A total of 116 differentially expressed transcription factors (TFs) were identified, and 17 TFs were found to belong to MYB families. Phylogenetic analysis revealed that these TFs may be involved in salt stress adaptation. Further analysis revealed that GmMYB46 was up-regulated by salt and mannitol and was localized in the nucleus. The salt tolerance of transgenic Arabidopsis overexpressing GmMYB46 was significantly enhanced compared to wild-type (WT). GmMYB46 activates the expression of salt stress response genes (P5CS1, SOD, POD, NCED3) in Arabidopsis under salt stress, indicating that the GmMYB46 protein mediates the salt stress response through complex regulatory mechanisms. This study provides information with which to better understand the molecular mechanism of salt tolerance in soybeans and to genetically improve the crop.

\section{INTRODUCTION}

Plant growth and development are often affected by unfavorable external stimuli such as drought, salinity, and extreme temperatures. Salt stress may lead to decreased crop yields and it reportedly affects approximately $6 \%$ of arable land and $20 \%$ of irrigated land (Chen et al., 2021a). Soil salinization affects plant growth 
primary stressor and immediately affects plant growth when the plant is exposed to high salinity. Ion toxicity occurs later when $\mathrm{NaCl}$ concentration has reached a threshold beyond which the ion homeostasis in plants is disrupted (van Zelm et al., 2020). High salinity stress inhibits plants' nutrient uptake, thus inhibiting growth and development (van Zelm et al., 2020). Salt stress stimulates the production of reactive oxygen species (ROS), such as hydrogen peroxide, superoxide, and hydroxyl radicals. ROS have a strong oxidative capacity and can cause cell plasma membrane damage, metabolic dysfunction, and cell death by disrupting redox homeostasis (Liang et al., 2018).

Plants have evolved many complex molecular and physiological mechanisms to adapt to salinized environments, such as the plant hormone signaling pathway, salt overly sensitive (SOS) signaling pathway, $\mathrm{Na}^{+}$efflux or sequestration in vacuoles, transcriptional regulation, improvement of antioxidant enzyme activity, and osmolyte (which include proline and soluble sugar) accumulation (van Zelm et al., 2020). Protein kinases (PKs) and protein phosphatases (PPs) in plant signal transduction pathways participate in abiotic stress responses through protein dephosphorylation. The PP2C protein family is reportedly important to the salt stress response because they affect plants' metabolic process, hormone levels, and growth factors (Wang et al., 2020a). In addition, the maintenance of intracellular sodium and potassium homeostasis is essential for the survival of plants under salt stress, and many ion channels, transporters, and antiporters play a role in this process. For example, HKT1 (high-affinity potassium transporter 1) was initially thought to be an $\mathrm{Na}^{+} / \mathrm{K}^{+}$ symporter in wheat, and that $\mathrm{Na}^{+}$was excreted by transpiration to enhance plant salt tolerance (Schachtman and Schroeder, 1994). However, the Arabidopsis AtHKT1 protein selectively transports $\mathrm{Na}^{+}$, affecting its distribution in roots and shoots (Ren et al., 2005). The tonoplast-localized NHX-type $\mathrm{Na}^{+} / \mathrm{H}^{+}$exchangers have been reported to sequestrate $\mathrm{Na}^{+}$into vacuoles and participate in $\mathrm{K}^{+}$homeostasis. For example, plasma membrane-localized NHX7/SOS1 flushes excess $\mathrm{Na}^{+}$through the roots and restricts excessive accumulation, which regulates intracellular ion homeostasis and enhances plant salt tolerance. However, the Arabidopsis sos 1 mutant showed more sensitivity to salt stress (Ji et al., 2013; Shi et al., 2002). Nieves-Cordones et al. (2010) found that the high-affinity potassium transporter 5 (HAK5) could promote $\mathrm{K}^{+}$absorption under high salinity conditions, which was beneficial to maintaining the balance of $\mathrm{Na}^{+} / \mathrm{K}^{+}$. However, some HAK family members can regulate $\mathrm{Na}^{+}$translocation. Wang et al. (2020b) revealed that S1HAK20 transported $\mathrm{Na}^{+}$and $\mathrm{K}^{+}$and 
55

regulated $\mathrm{Na}^{+} / \mathrm{K}^{+}$balance under salt stress. The mechanism for transcriptional regulation of plant salt tolerance has attracted much attention in the scientific community. Transcription factors (TFs) are considered to be gene switches, which activate or inhibit the expression of downstream stress-related genes by binding to specific cis-acting elements on promoters and forming a complex gene regulatory network to alleviate the damage caused by abiotic stress (Hoang et al., 2017). Many TFs are involved in regulating the response and adaptation of plants to salt stress, including WRKY (Li et al., 2019a), MYB (Du et al., 2018), AP2/ERF (Zhao et al., 2019a), NAC (Li et al., 2021). Plants respond to osmotic stress by accumulating a large number of osmolytes under high salinity conditions, these osmolytes generally include proline, sugar alcohols, sorbitol, and quaternary ammonium compounds (van Zelm et al., 2020). Liu et al. (2015) found that the overexpression of AtbHLH112 in Arabidopsis activated the expression of P5CS and inhibited the expressions of the P5CDH and ProDH genes, thus increasing proline synthesis while reducing its degradation. The increased proline content contributes to better abiotic stress tolerance of Arabidopsis.

Soybean (Glycine max L.) is a global staple edible oil and food crop, which also provides the raw material for the manufacture of animal feeds. Salt stress adversely affects the growth of soybean plants, which leads to reduced yields. At present, many key genes related to salt tolerance have been identified in soybeans. The CHX-type ion transport protein GmSALT3 improves the salt tolerance of soybean plants by promoting $\mathrm{Na}^{+}$ and $\mathrm{Cl}^{-}$exclusion (Qu et al., 2021). The salt tolerance of soybean hairy roots and transgenic Arabidopsis overexpressing $\mathrm{GmCHX} 20$ a decreases, promoting the absorption and accumulation of $\mathrm{Na}^{+}$in roots be due to GmCHX20a, while GmCHX1 (GmSALT3) was shown to protect transgenic Arabidopsis plants via $\mathrm{Na}^{+}$ exclusion under salt treatments (Jia et al., 2020). The expression level of the MYB transcription factor GmMYB84 was up-regulated under salt stress, and the overexpression of GmMYB84 enhanced the salt tolerance of transgenic soybeans (Zhang et al., 2020a). Salt-induced soybean NAC transcription factor, GmSIN1, promoted root growth and enhanced salt tolerance by controlling abscisic acid production and ROS (Li et al., 2019b). However, as there are limited genetic resources to improve soybean genetics these require further study.

Understanding the molecular mechanisms of plant salt tolerance is a prerequisite for the development of salt-tolerant crop varieties, and technological advances can help to clarify the complex mechanisms of plant 
82

response to salt stress. Transcriptome analysis is a powerful tool to uncover stress-related regulatory networks in plants. It is widely used to identify stress-responsive genes and genetic control elements. A large number of salt-induced genes have been identified in oilseed rape (Yong et al., 2014), rice (Zhou et al., 2016), watermelon (Song et al., 2020a), kenaf (Munsif et al., 2020), and maize (Chen et al., 2021b). Ali et al. (2012) found that soybeans responded to salt stress through abscisic acid (ABA) biosynthesis based on tag sequence data combined with GO analysis. Zhao et al. (2019a) identified differentially expressed genes through transcriptome analysis, including Glyma.02G228100, Glyma.03G226000, Glyma.03G031000, Glyma.03G031400, Glyma.04G180300, Glyma.04G180400, Glyma.05G204600, Glyma.08G189600, Glyma.13G042200, and Glyma.17G17320, which were considered to be the key genes involved in the salt tolerance mechanism in salt-tolerant soybeans. It is generally believed that plants sense salt stress mainly through their roots, but high salinity may lead to physiological changes such as growth inhibition, leaf wilting, excessive accumulation of ROS in leaves, and increased $\mathrm{Na}^{+}$content. However, the molecular mechanism of plant shoots (especially leaves) in response to salt stress is not well-established. In this study, the shoot (including stems and leaves) of soybean seedlings was used for transcriptome sequencing (RNA sequencing, RNA-seq), and Kyoto Encyclopedia of Genes and Genomes (KEGG) enrichment combined with other bioinformatics methods were used to identify key pathways and genes induced by salt stress. This study will provide data for the investigation of molecular mechanisms of the salt response in soybeans and cultivation of salt-resistant varieties.

\section{MATERIALS AND METHODS}

\section{Plant growth and salt treatment}

Cultivated soybean seeds (Tianlong No. 1) were sterilized with $75 \%$ ethanol for 10 min, washed with sterile distilled water, and laid on wet filter paper for two days. The germinated seeds were transferred to quartz sand and irrigated with Hoagland nutrient solution, then cultured in a plant growth chamber ( $16 \mathrm{~h}$ day $/ 8$ h night, $25 \pm 2^{\circ} \mathrm{C}, 60-70 \%$ relative humidity). After $10 \mathrm{~d}$, soybean seedlings with consistent growth were selected and divided into two groups: seedlings irrigated with Hoagland nutrient solution as control, and those irrigated with Hoagland nutrient solution containing $120 \mathrm{mM} \mathrm{NaCl}$ as the treatment. The shoots (stems and leaves) were obtained after being treated for $6 \mathrm{~h}$ and were frozen in liquid nitrogen and stored at $-80^{\circ} \mathrm{C}$ for 
109

110

111

112

113

114

115

116

117

118

119

120

121

122

123

124

125

126

127

128

129

130

131

132

133

134

135

transcriptome sequencing. The control group and treatment group each contained three biological replicates.

Phenotypic comparison and physiological measurements

Histochemical staining with NBT (Nitrotetrazolium blue chloride) and DAB (3, 3'-Diaminobenzidine) was used to examine the $\operatorname{ROS}\left(\mathrm{O}_{2}{ }^{-}\right.$and $\left.\mathrm{H}_{2} \mathrm{O}_{2}\right)$ accumulation levels in the leaves (He et al., 2019). After $0 \mathrm{~h}, 3 \mathrm{~h}$, $6 \mathrm{~h}$, and $12 \mathrm{~h}$ salt treatment, the leaves were removed and immersed in DAB and NBT staining solution. Chlorophyll was removed with alcohol for photography after $12 \mathrm{~h}$ at room temperature. After 6 days of salt treatment, plant phenotypes were analyzed and compared, and physiological indexes related to salt stress (including plant height, fresh weight per plant, proline content, $\mathrm{Na}^{+}$, and $\mathrm{K}^{+}$ion content) were determined as previously reported (Zhang et al., 2020b).

\section{RNA isolation, cDNA library preparation, and Illumina sequencing}

The total RNA was isolated from soybean shoot samples using a mirVana miRNA Isolation Kit (Invitrogen, AM1561) according to the manufacturer's protocol. The contained DNA was digested with DNase

I. The quality and concentration of RNA was detected using Agarose gel electrophoresis and Nanodrop 2000 (ThermoFisher Scientific, Massachusetts, USA), respectively. The mRNA in the total RNA was enriched using magnetic beads with Oligo (dT), and approximately $4 \mu \mathrm{g}$ mRNA was collected from each sample for library construction using a TruSeq Stranded mRNA LTSample Prep Kit (Illumina, San Diego, USA) following the manufacturer's protocol. The libraries were then sequenced on the Illumina sequencing platform (Illumina HiSeqTM 2500). The raw reads generated in this study were deposited in the NCBI database under accession number PRJNA741583.

\section{Validation by qRT-PCR analysis}

The expression of 10 randomly selected genes was evaluated by qRT-PCR analysis to validate the transcriptome data. This selection included 5 up-regulated genes (LOC100807235, LOC100816551, LOC100785783, LOC100787314, LOC100795929) and 5 down-regulated genes (LOC100805378, LOC102663255, LOC100306125, LOC100787705, LOC100819491). qRT-PCR was conducted using Bio-Rad CFX96 PCR System (USA, Bio-Rad) with a $20 \mu \mathrm{L}$ reaction mixture. The mixture consisted of $10 \mu \mathrm{L}$ HieffTM qPCR SYBR® Green Master Mix (No Rox Plus) (11201ES, Yeasen, Shanghai, China), $0.5 \mu \mathrm{L}(10 \mu \mathrm{M})$ Primer F, $0.5 \mu \mathrm{L}(10 \mu \mathrm{M})$ Primer $\mathrm{R}$, and $9 \mu \mathrm{L}(100 \mathrm{ng} / \mu \mathrm{L})$ diluted cDNA. Relative expression of the genes was 
analyzed with the $2^{-\Delta \Delta \mathrm{Ct}}$ method using the $G m E F-1 \alpha$ gene as an internal control to normalize the level of gene expression (Zhao et al., 2020). The primers for qRT-PCR analysis were designed using the Primer Blast tool on the NCBI (http://www.ncbi.nlm.nih.gov/). All primers used in this study are shown in Table S1.

\section{RNA-Seq analysis}

The transcriptome sequencing was conducted by OE Biotech Co., Ltd. (Shanghai, China). Raw reads containing ploy-N and the low-quality reads were filtered to obtain the clean reads using Trimmomatic software (Bolger et al., 2014). The clean reads were mapped to the cultivated soybean (Glycine max (Linn.) Merr.) genome (https:/www.ncbi.nlm.nih.gov/genome/5?genome_assembly_id=401179). The Q20, Q30, and GC content from the clean read data were calculated and used to evaluate all samples' sequencing quality. All further analyses were based on high-quality data from clean reads. Pearson correlation analysis was performed to identify the repeatability of three biological replicates in the same group.

The gene expression levels were normalized to fragments per kilobase of transcript per million fragments mapped (FPKM) using Cufflinks v2.2.2 software. Differentially expressed genes (DEGs) were identified by comparing the read counts of the control and salt-treated samples' gene transcripts using the DESeq R package function's estimate size factors and nbinom tests (Anders \& Huber, 2010). The p-value $<0.05$ and $\mid \log 2$ fold change $\mid>1$ or $p$-value $<0.01$ and $\mid \log 2$ fold change $\mid>1.5$ were set as the threshold for significantly differential expression (Long et al., 2019).

KEGG pathways' enrichment of DEGs was determined using KOBAS software with FDR $<0.01$. All of the genes annotated as transcription factors in DEGs were screened to identify the response of transcription factors to salt stress in soybean shoots.

\section{Phylogenetic analysis of MYB family members}

Some members of the MYB families reportedly involved in plant abiotic stress were retrieved from the NCBI database, and phylogenetic analysis was conducted with the corresponding family members in the salttreated soybeans' DEGs. The phylogenetic tree was constructed using the neighbor-joining (NJ) method with 1,000 bootstrap replicates in MEGA X (Zhang et al., 2018). The FPKM values of 17 differentially expressed MYBs were used to draw the heatmap using TBtools (v1.0983) (Chen et al., 2020).

\section{Expression pattern and subcellular localization of GmMYB46}


Ten-day old soybean seedlings were treated with $120 \mathrm{mM} \mathrm{NaCl}$ or $300 \mathrm{mM}$ Mannitol. The leaves were removed at $0,3,6$, and $12 \mathrm{~h}$, quick-frozen with liquid nitrogen, and stored at $-80^{\circ} \mathrm{C}$ for RNA extraction and detection of GmMYB46 expression pattern using semi-quantitative PCR. The CDS removed the stop codon of GmMYB46, which was cloned and ligated into the pCAMBIA1300-GFP vector to obtain a fusion expression vector GmMYB46:eGFP. This vector was introduced into Agrobacterium tumefaciens GV3101 before the positive A.tumefaciens was injected into Nicotiana benthamiana leaves. Plants were cultured for $2 \mathrm{~d}$. The GFP fluorescence was observed and imaged using a laser confocal microscope (Zeiss LSM880 Meta, Jena, Germany).

\section{Salt tolerance analysis of transgenic Arabidopsis}

The full-length CDS sequence of GmMYB46 was cloned to construct the overexpressed vector 35S:GmMYB46 and the wild-type Arabidopsis Col-0 was transformed using the floral dip method by Agrobacterium-mediated. The transgenic lines were identified by hygromycin resistance screening and semiquantitative PCR. The seeds were evenly sown on nutrient-rich soil, kept in the dark at $4^{\circ} \mathrm{C}$ for $3 \mathrm{~d}$, and then placed in a growth chamber for $10 \mathrm{~d}$. The control group (CK) was irrigated with deionized water for culturing, and the treatment group $(\mathrm{NaCl})$ was irrigated with $150 \mathrm{mM} \mathrm{NaCl}$ solution. The phenotype was observed and photographed after $5 \mathrm{~d}$ and $7 \mathrm{~d}$ of treatment, and physiological indicators (survival rate, relative water content, malondialdehyde (MDA) content, proline content, SOD, and POD activities) were measured on day seven, following previously reported methods (Chen et al., 2019; He et al., 2019). The leaves were removed for RNA extraction $12 \mathrm{~h}$ after salt treatment, qRT-PCR analysis of salt stress-related genes was performed, and AtACTIN2 was used as an internal reference using the same methods as noted above.

\section{Statistical analysis}

All data were statistically analyzed using IBM SPSS Statistics software (v20.0, NY, USA). Means \pm SD are shown in this study $(\mathrm{n} \geq 3)$. The Student's t-test was used to calculate P-values $(\mathrm{P}<0.05)$ to determine the significance of the differences among treatments.

\section{RESULTS}

\section{Salt injury in soybean shoots exposed to high salinity}

To observe the effect of salt stress on the growth of soybean shoots, 10-day-old soybean seedlings were 
selected for salt treatment. NBT and DAB staining results showed that the contents of $\mathrm{H}_{2} \mathrm{O}_{2}$ and $\mathrm{O}_{2}{ }^{-}$in leaves were significantly higher than those at $0 \mathrm{~h}$ and $3 \mathrm{~h}$ after salt treatment for $6 \mathrm{~h}$ and $12 \mathrm{~h}$ (Fig. 1A), indicating that excessive ROS had been generated and accumulated in plant leaves at $6 \mathrm{~h}$ salt treatment. Therefore, the $6 \mathrm{~h}$ salt-treated plants were used for transcriptome sequencing. The phenotypic comparison showed that the growth of soybean seedlings after $6 \mathrm{~d}$ of salt treatment was significantly inhibited, and plant height and fresh weight were lower than those of the control (CK) (Fig. 1B, C, D). In addition, the content of $\mathrm{Na}^{+}$in shoots of salttreated soybean seedlings increased significantly and $\mathrm{K}^{+}$decreased compared with CK (Fig. 1E, F). Compared with CK, a large amount of proline accumulated in the shoot of the salt-treated soybean (Fig. 1G).

\section{Transcriptome analyses}

RNA-Seq analysis was performed on seedlings treated with $0 \mathrm{mM}(\mathrm{CK})$ and $120 \mathrm{mM} \mathrm{NaCl}$ for $6 \mathrm{~h}$. We constructed six libraries from the control and $\mathrm{NaCl}$ treatment groups (three biological replicates in each group) for analysis using RNA-Seq. These libraries yielded 50.05 to 58.13 million raw reads, and 48.85 to 56.88 million clean reads (Table 1). The Q30 in each library was greater than $92 \%$, indicating that the quality of the sequencing data could be used for further analysis. Approximately $98 \%$ of the clean reads were mapped to the soybean genome. Correlation analysis revealed that the relationships among the three biological replicates in the same group were more closely related (Fig. 2A). The volcano plot showed that 466 genes were upregulated and 769 genes were down-regulated after salt treatment based on the loose DEGs restrictions $(\mathrm{p}<$ 0.05, $\mid \log 2$ fold change $\mid>1$ ) (Fig. 2B, Table S2). However, stricter parameters revealed ( $p<0.01, \mid \log 2$ fold change $\mid>1.5$ ) 425 DEGs, including 127 up-regulated and 298 down-regulated genes (Table S3). Transcriptome data was verified by qRT-PCR analysis and showed that the fold variation between RNA-seq expression and qRT-PCR analyses were linearly correlated $\left(\mathrm{R}^{2}=0.8568\right)$ (Fig. $\left.2 \mathrm{C}\right)$.

KEGG enrichment analysis of up- and down-regulated DEGs was conducted using the KOBAS database. The top 20 most highly enriched pathways were displayed (Fig. 3). The up-regulated DEGs induced by salt treatment were significantly enriched in 'Plant hormone signal transduction' (34), 'Phenylalanine metabolism' (4), 'Fructose and mannose metabolism' (6), 'Carotenoid biosynthesis' (4), and 'MAPK signaling pathway' (8) (Fig. 3A). The down-regulated DEGs were significantly enriched in 'Plant hormone signal transduction' (25), 'Ribosome biogenesis in eukaryotes' (9), 'MAPK signaling pathway' (13), 'Plant-pathogen interaction' (15), 
217 and 'Arginine and proline metabolism' (6) (Fig. 3B).

218 Key genes in early response to salt stress

219 The up- and down-regulated DEGs can be divided into the following five categories in the KEGG

220

221

222

223

224

225

226

227

228

229

230

231

232

233

234

235

236

237

238

239

240

241

242

243

database: Cellular Processes, Environmental Information Processing, Genetic Information Processing, Metabolism, and Organismal Systems. Among them, the number of up-regulated and down-regulated DEGs is the largest in signal transduction categories, which belong to Environmental Information Processing, and include 34 and 29 genes, respectively (Table 2). The 34 up-regulated genes contain hormone-related proteins and PP2C family members, while down-regulated genes mainly include the ABA-related genes and probable xyloglucan endotransglucosylase/hydrolase protein family members (Table S4). Environmental adaptation categories include four up-regulated genes and 15 down-regulated genes (Table 2, Table S5). Furthermore, seven genes from the ABA receptors PYL family enriched in both the plant hormone signal transduction pathway and the MAPK signaling pathway were significantly down-regulated, including LOC100788576, LOC100819216, LOC100804400, LOC100805378, LOC100792229, LOC100798803, and LOC100788199 (Fig. 4A, E). The PP2C (Phosphatase 2C) family genes downstream of PYL were significantly up-regulated and include LOC100793293, LOC100803764, LOC100807235, LOC100818568, LOC100776356, LOC100796161, and LOC100781388 (Fig. 4B, F). The expression levels of ABF family members downstream of SNF1-related protein kinase 2 (SNRK2), LOC100819313 and GmbZIP1, were significantly up-regulated (Fig. 4A). Proline dehydrogenase genes (PDH, LOC100797464, and LOC100799876) in the arginine and proline metabolic pathways were significantly down-regulated (Fig. 4C, G) The phenylalanine ammonia-lyase genes (GmPAL1.2, GmPAL1.3, GmPAL2.2, and GmPAL2.4) in the phenylalanine metabolism pathway were significantly up-regulated to promote the production of cinnamic acid (Fig. 4D, H), which is important for salt stress adaptation.

\section{Transcription factors (TFs) involved in salt stress}

The differentially expressed TFs in DEGs were statistically analyzed, as shown in Fig. 5. We found that 1,235 DEGs contained a total of 116 transcription factor coding genes, accounting for $9.4 \%$ of DEGs. Of these, 49 were up-regulated and 67 were down-regulated. There were a large number of AP2/ERF, MYB, and bHLH families with 32, 17, and 14 differentially expressed TFs, respectively. Further analysis showed that the MYB 
244 family had the most members (11) in the up-regulated TFs, while the AP2/ERF family had the most members

245 (26) in the down-regulated TFs. We searched MYB proteins that were reportedly involved in plant abiotic 246 stress and conducted the phylogenetic analysis with MYB members in soybean DEGs. As shown in Fig. 6A, 247 the up-regulated LOC778071 and SlMYB102 (Solyc02g079280) (Zhang et al., 2020c) were highly homologous 248 and in the same clade; LOC100799410, MdMYB46 (XP_008363629) (Chen et al., 2019), and TaMYB86B 249 (KM066946) (Song et al., 2020a) were in the same clade, and LOC100805341 and OsMYB2 (AK120551) 250 (Yang et al., 2012) were in the same clade. Further analysis showed that the expression level of

GmMYB46 was up-regulated by salt treatment and localized in the nucleus

Semi-quantitative PCR analysis showed that the expression level of GmMYB46 was significantly increased in leaves treated with salt for 6 and $12 \mathrm{~h}$, while the expression of GmMYB46 first increased (6 h) and then decreased $(12 \mathrm{~h}$ ) under mannitol treatment (Fig. 6C). In addition, the GFP fusion vector GmMYB46:eGFP was constructed and the transient expression was carried out in tobacco leaves. The results showed that green fluorescence was only observed in the nucleus of cells of GmMYB46:GFP transformed leaves, indicating that GmMYB46 protein was localized in the nucleus (Fig. 6D).

\section{Overexpression of GmMYB46 enhances the salt tolerance of transgenic Arabidopsis}

An overexpression vector (35S:GmMYB46) was constructed to explore the function of GmMYB46 in plant salt tolerance, and transgenic Arabidopsis lines (OX46.1, OX46.2) were obtained (Fig. S1A, B). Phenotypic analysis showed that the growth of Arabidopsis seedlings was significantly inhibited by $150 \mathrm{mM} \mathrm{NaCl}$ treatment for $5 \mathrm{~d}$ and $7 \mathrm{~d}$, but OX46.1 and OX46.2 plants were less affected by salt than WT (Col-0) (Fig. 7A). The survival rates of OX46.1 and OX46.2 were significantly higher than those of the WT after 5 and $7 \mathrm{~d}$ of salt treatment (Fig. 7B). In addition, the relative water content of OX46.1 and OX46.2 plants was significantly higher than that of WT (Fig. 7C), while the MDA content was significantly lower than that of WT under salt stress (Fig. 7D). The proline content and the activities of SOD and POD increased significantly, while the overexpressed lines were significantly higher than that of WT (Fig. 7E, F). In addition, we examined the 
271

272

273

274

275

276

277

278

279

280

281

282

283

284

285

286

287

288

289

290

291

292

293

294

295

296

297

expression level of P5CS1, a key gene for proline synthesis, was significantly up-regulated in Col-0, OX46.1, and OX46.2 plants induced by salt, and it was significantly higher in overexpressed lines than in WT (Fig. 8A). Antioxidant enzyme synthesis-related genes $S O D, P O D$, and $N C E D 3$, a key gene for ABA biosynthesis, also showed a salt-induced expression pattern similar to P5CS1 (Fig. 8B, C, D).

\section{DISCUSSION}

Salt stress is one of the major environmental factors limiting plant growth and productivity. Plants have evolved complex physiological, biochemical, and molecular regulatory mechanisms to adapt to adverse conditions. These involve the expression changes of a large number of regulatory genes. Transcriptome sequencing is an effective tool for comprehensive analysis at the transcriptional level for plants under abiotic stress (Zhang et al., 2020b). There have been many studies on the transcriptome analysis of legumes using RNA-seq, including medicago (Shu et al., 2018), glycine (Sun et al., 2016), common bean (Hiz et al., 2014), and Arachis hypogaea (Zhang et al., 2020b). Sun et al. (2016) performed small RNA transcriptome analysis on root tip tissues of normal and salt-treated soybean seedlings and identified 66 salt-responsive miRNAs, and then demonstrated that salt-induced miR399 played an important role in regulating the developmental plasticity of soybean roots. Plant roots are known to directly encounter salinized soil as the first line of defense; accordingly, most studies have focused on the molecular regulatory mechanisms of the root response to salt stress (Zhang et al., 2016; Zhao et al., 2020). However, these molecular mechanisms and regulatory networks have not been studied in plant shoots when growth is inhibited due to salt stress. Zeng et al. (2019) performed transcriptome sequencing on salt-treated soybean leaves, however only eight genes were preliminarily identified, and the function of these genes has not been explored in plants in their report. In contrast to previous work, shoot tissues (including stems and leaves) of salt-treated soybean seedlings were used for transcriptome sequencing in this study, which may better reflect the response of the above-ground parts of soybeans to salt stress. More importantly, transcriptome and bioinformatics analysis combined with Arabidopsis genetic transformation technique were used to preliminarily demonstrate the function of GmMYB46 in response to salt stress in our work.

We identified some important pathways and key candidate genes that may be involved in the salt stress response among 1,235 DEGs. Wu et al. (2017) conducted KEGG enrichment analysis of salt-induced DEGs 
298

299

300

301

302

303

304

305

306

307

308

309

310

311

312

313

314

315

316

317

and found 'Plant hormone signal transduction', 'MAPK signaling pathway', 'Arginine and proline metabolism' and other secondary metabolite pathways were significantly enriched. Tian et al. (2018) used KEGG analysis to determine that 'Plant hormone signal transduction pathway' was significantly enriched in both salt-sensitive and salt-tolerant Rosa chinensis. Similarly, our analysis found that DEGs were significantly enriched in 'Plant hormone signal transduction', 'MAPK signaling pathway', 'Phenylalanine metabolism', 'Arginine and proline Metabolism' pathways (Fig. 3). These pathways play an important role in the process of plant salt stress response and the results provide a reference for salt tolerance genes in soybeans and assist in analyzing molecular regulatory mechanisms.

$\mathrm{ABA}$ is one of the most important salt stress hormones. It plays a crucial role in salt stress defense. ABA is involved in the regulation of osmotic stress, ion homeostasis, and the scavenging of reactive oxygen species induced by salt injury (Yu et al., 2020). It is widely believed that PYR/PYL protein, PP2Cs, and SnRK2 kinase constitute the core module of ABA signaling (PYL-PP2CS-SnRK2s), which is responsible for the earliest ABA signal recognition and transduction in plants (Gong et al., 2020). Liu et al. (2012) showed that overexpression of AtPP2CG1 enhanced the salt tolerance of transgenic Arabidopsis by activating the expression of salt stress response genes $R D 29 A, R D 29 B, D R E B 2 A$, and KIN1. In this study, we found that the ABA receptor PYL family genes significantly enriched in 'Plant hormone signal transduction' and 'MAPK signaling pathway' were significantly down-regulated, while PP2C family members were significantly upregulated. Two downstream ABF family genes (LOC100819313 and GmbZIP1) were also significantly upregulated (Fig. 4). Lei et al. (2021) conducted transcriptome sequencing of salt-treated Ricinus communis L. and found that $P P 2 C$ genes were up-regulated in salt-treated cultivated $R$. communis, which inhibited the activity of SnRK2 kinase and further promoted the up-regulation of downstream ABF genes. The results in this work are consistent with previous reports, further confirming the function of the ABA signaling pathway PYLPP2CS-SnRK2s in short-term salt stress. In addition, previous reports have shown that proline metabolism is an important pathway in response to oxidative stress caused by salt injury, and that the accumulation of proline is protective for plants (Verbruggen \& Hermans, 2008). In this study, three key genes of the proline degradation pathway (PDH, LOC100797464, LOC100799876) were significantly down-regulated (Fig. 4), which reduce the degradation of proline in shoots and increase its accumulation in response to salt stress. 
325 Phenylalanine ammonia-lyase (PAL) is a key enzyme in the phenylalanine pathway that catalyzes the

326

327

328

329

330

331

332

333

334

335

336

337

338

339

340

341

342

343

344

345

346

347

348

349

350

351 deamination of phenylalanine to produce cinnamic acid. Some PAL genes (Aradu.IU1HH, Araip. 69J63, Araip.GM19P, and Araip.V9S7Z) in peanuts were significantly up-regulated under salt stress (Wanner et al., 1995; Zhang et al., 2020b). Singh et al. (2013) showed that cinnamic acid could reduce the accumulation of ROS in maize plants by providing ROS-scavenging enzymes under salt stress. We found that the expression levels of PAL family genes (GmPAL1.2, GmPAL1.3, GmPAL2.2, GmPAL2.4) were also significantly upregulated in salt-treated soybeans (Fig. 4), indicating that these genes were involved in the response to salt stress. However, the means by which these genes exert their regulatory functions requires further study.

TFs are key regulatory factors in the complex signal network of plants in response to abiotic stress. The function of several TFs in the regulation of salt stress in plants has been reported, including that of AP2/ERF, MYB, bHLH, WRKY, and C2H2 (Ciftci-Yilmaz et al., 2007; Li et al., 2019a; Liu et al., 2015; Makhloufi et al., 2014; Zhao et al., 2019b). In this study, 116 TFs were found in the differential genes by RNA-seq, accounting for $9.4 \%$ of the DEGs. These TFs were distributed among different families, including AP2/ERF, bZIP, NAC, MYB, BHLH, C3H, C2H2, HD-ZIP, and WRKY, etc. The AP2/ERF family has the largest number of members (32), followed by MYB (17) and bHLH (14) (Fig. 5). This is consistent with transcriptome analysis results from other species undergoing salt treatment. Long et al. (2019) found that the number of the AP2/ERF family members was largest among the DEGs treated with salt, accounting for 17.6\%. Further analysis showed that MYB had the most members (11) in the up-regulated TFs (Fig. 5). Munsif et al. (2020) also found that the MYB family has the largest amount of up-regulated differentially expressed TFs in salt-treated isonuclear kenaf. Zhang et al. (2020c) showed that overexpression of SIMYB102 enhanced salt tolerance of transgenic tomatoes by regulating a series of molecular and physiological processes. OsMYB2-overexpressing transgenic rice plants were more tolerant than wild-type to salt stress (Yang et al., 2012). Chen et al. (2019) showed that MdMYB46 can enhance the salt tolerance of apples by activating stress-related genes (MdABRE1A, MdDREB2a, MdRD22, and MdRD29A). TaMYB86B affects wheat's salt tolerance by regulating ion homeostasis, maintaining osmotic balance, and reducing ROS levels (Song et al., 2020b). Phylogenetic analysis revealed that GmMYB46 (LOC100799410), MdMYB46, and TaMYB86B were highly homologous in the same clade (Fig. 6A). Analysis also showed that the expression level of the GmMYB46 gene was the 
352 highest up-regulated expression among all differentially expressed MYBs (Fig. 6B). Therefore, we believe that

353 it is worth exploring the function of GmMYB46 under salt stress.

354 Further analysis revealed that the expression level of GmMYB46 first increased and then decreased under the 355 treatment of mannitol compared with salt treatment (Fig. 6C). Plants first go through a rapid osmotic stress 356 phase when exposed to salt in the environment before experiencing ion toxicity caused by salt stress (Zhao et al., 2019a). Mannitol treatment led to osmotic stress. The expression pattern of GmMYB46 suggesting that it may function in the early response to osmotic stress, and its longer response time to salt treatment indicated that it may play an important role in salt stress. The salt tolerance of Arabidopsis overexpressing GmMYB46 is significantly enhanced at $5 \mathrm{~d}$ and $7 \mathrm{~d}$ after salt treatment (Fig. 7). These results implied that GmMYB46 plays an important role in both short-term salt response and long-term salt stress tolerance. Chen et al. (2019) reported similar results and found that a short-term salt responsive gene, MdMYB46, in apples plays an important role in enhancing the long-term salt stress tolerance of transgenic apples. MDA content, as a product of membrane lipid peroxidation, reflects the degree of damage of plant cells due to abiotic stress, including salt stress (Song et al., 2019). We found that the MDA content of overexpressed lines under salt stress was significantly lower than that of WT (Fig. 7). The enhanced activity of SOD and POD helps plants reduce oxidative damage (Zhang et al., 2020d) and the proline content may reflect the stress resistance of plants. We found that the activity of SOD and POD, and the content of proline were significantly increased in overexpression lines compared with WT under salt stress (Fig. 7). In addition, the key genes for proline biosynthesis P5CS1 (Liu et al., 2015), and antioxidant enzymes SOD and POD encoding genes, were significantly up-regulated in the overexpression lines after salt treatment compared with WT (Fig. 8). NCED3, as a key gene in ABA synthesis (van Zelm et al., 2020), was significantly upregulated under salt treatment, and the upregulated ratio in the overexpressed line was significantly higher than that in WT. These results further suggest that the regulation of GmMYB46 in the salt stress response is complex, and the detailed molecular regulatory mechanism requires additional study.

\section{CONCLUSIONS}

The shoots of control and salt-treated soybean seedlings were used for transcriptome sequencing to analyze their molecular mechanisms in response to salt stress. Transcriptome analysis revealed a total of 1,235 
379

380

381

382

383

384

DEGs, of which 466 genes were up-regulated and 769 genes were down-regulated. The DEGs involved in important pathways were determined by KEGG enrichment, which included 'Plant hormone signal transduction', 'MAPK signaling pathway', 'Arginine and proline metabolism', and 'Phenylalanine metabolism'. We further analyzed the DEGs in these pathways to characterize their possible functions in responses to salt stress as well as TFs related to salt stress responses. The phylogenetic trees of differentially expressed MYBs induced by salt stress were constructed. The salt tolerance of transgenic Arabidopsis overexpressing GmMYB46 was significantly enhanced, and GmMYB46 was found to activate the expression of salt stress response genes (P5CS1, SOD, POD, NCED3) in Arabidopsis under salt stress. This work will provide a basis for future studies to discover novel salt-tolerance genes, further investigate the molecular mechanism of salt tolerance in soybeans, and cultivate strong salt resistant varieties.

\section{REFERENCES}

Ali Z, Zhang DY, Xu ZL, Xu L, Yi JX, He XL, Huang YH, Liu XQ, Khan AA, Trethowan RM, and Ma HX. 2012. Uncovering the salt response of soybean by unraveling its wild and cultivated functional genomes using tag sequencing. PLoS One 7:e48819. 10.1371/journal.pone.0048819

Anders S, and Huber W. 2010. Differential expression analysis for sequence count data. Genome Biology 11: R106.

Bolger AM, Lohse M, and Usadel B. 2014. Trimmomatic: a flexible trimmer for Illumina sequence data. Bioinformatics 30:2114-2120.

Chen C, Chen H, Zhang Y, Thomas HR, Frank MH, He Y, and Xia R. 2020. TBtools: An integrative toolkit developed for interactive analyses of big biological data. Mol Plant 13:1194-1202. 10.1016/j.molp.2020.06.009

Chen KQ, Song MR, Guo YN, Liu LF, Xue H, Dai HY, and Zhang ZH. 2019. MdMYB46 could enhance salt and osmotic stress tolerance in apple by directly activating stress-responsive signals. Plant Biotechnol $J$ 17:2341-2355. 10.1111/pbi.13151

Chen XX, Ding YL, Yang YQ, Song CP, Wang BS, Yang SH, Guo Y, and Gong ZZ. 2021a. Protein kinases in plant responses to drought, salt, and cold stress. Journal of Integrative Plant Biology 63:53-78. $10.1111 /$ jipb.13061 
406

407

408

Chen MX, Lu CC, Sun PC, Nie YX, Tian Y, Hu QJ, Das D, Hou XX, Gao B, Chen X, Liu SX, Zheng CC, Zhao XY, Dai L, Zhang JH, and Liu YG. 2021b. Comprehensive transcriptome and proteome analyses reveal a novel sodium chloride responsive gene network in maize seed tissues during germination. Plant Cell and Environment 44:88-101. 10.1111/pce.13849

Ciftci-Yilmaz S, Morsy MR, Song LH, Coutu A, Krizek BA, Lewis MW, Warren D, Cushman J, Connolly EL, and Mittler R. 2007. The EAR-motif of the Cys2/His2-type zinc finger protein ZAT7 plays a key role in the defense response of Arabidopsis to salinity stress. Journal of Biological Chemistry 282:9260-9268. 10.1074/jbc.M611093200

Du YT, Zhao MJ, Wang CT, Gao Y, Wang YX, Liu YW, Chen M, Chen J, Zhou YB, Xu ZS, and Ma YZ. 2018. Identification and characterization of GMMYB118 responses to drought and salt stress. BMC Plant Biol 18:320. 10.1186/s12870-018-1551-7

Gong Z, Xiong L, Shi H, Yang S, Herrera-Estrella LR, Xu G, Chao DY, Li J, Wang PY, Qin F, Li J, Ding Y, Shi Y, Wang Y, Yang Y, Guo Y, and Zhu JK. 2020. Plant abiotic stress response and nutrient use efficiency. Sci China Life Sci 63:635-674. 10.1007/s11427-020-1683-x

He F, Li HG, Wang JJ, Su Y, Wang HL, Feng CH, Yang Y, Niu MX, Liu C, Yin W, and Xia X. 2019. PeSTZ1, a $\mathrm{C} 2 \mathrm{H} 2$-type zinc finger transcription factor from Populus euphratica, enhances freezing tolerance through modulation of ROS scavenging by directly regulating PeAPX2. Plant Biotechnol J 17:2169-2183. $10.1111 /$ pbi. 13130

Hiz MC, Canher B, Niron H, and Turet M. 2014. Transcriptome analysis of salt tolerant common bean (Phaseolus vulgaris L.) under saline conditions. PLoS One 9:e92598. 10.1371/journal.pone.0092598

Hoang XLT, Nhi DNH, Thu NBA, Thao NP, and Tran LSP. 2017. Transcription factors and their roles in signal transduction in plants under abiotic stresses. Current Genomics 18:483-497. $10.2174 / 1389101918666170227150057$

Ji HT, Pardo JM, Batelli G, Van Oosten MJ, Bressan RA, and Li X. 2013. The Salt Overly Sensitive (SOS) Pathway: established and emerging roles. Molecular Plant 6:275-286. 10.1093/mp/sst017

Jia Q, Li MW, Zheng C, Xu Y, Sun S, Li Z, Wong FL, Song J, Lin WW, Li Q, Zhu Y, Liang K, Lin W, and Lam HM. 2020. The soybean plasma membrane-localized cation $/ \mathrm{H}^{+}$exchanger GmCHX20a plays a 
negative role under salt stress. Physiol Plant. 10.1111/ppl.13250

434

Lei P, Liu Z, Hu YB, Kim H, Liu S, Liu JQ, Xu LP, Li JX, Zhao Y, Yu ZL, Qu YT, Huang FL, and Meng FJ. 2021. Transcriptome analysis of salt stress responsiveness in the seedlings of wild and cultivated Ricinus communis L. Journal of Biotechnology 327:106-116. 10.1016/j.jbiotec.2020.12.020

Li M, Chen R, Jiang QY, Sun XJ, Zhang H, and Hu Z. 2021. GmNAC06, a NAC domain transcription factor enhances salt stress tolerance in soybean. Plant Molecular Biology 105:333-345. 10.1007/s11103-02001091-y

Li Z, Li L, Zhou KH, Zhang YH, Han X, Din YP, Ge XY, Qin WQ, Wang P, Li FG, Ma ZY, and Yang ZE. 2019. GhWRKY6 acts as a negative regulator in both transgenic Arabidopsis and cotton during drought and salt stress. Frontiers in Genetics 10:392. 10.3389/fgene.2019.00392

Li S, Wang N, Ji DD, Zhang WX, Wang Y, Yu YC, Zhao SZ, Lyu MH, You JJ, Zhang YY, Wang LL, Wang XF, Liu ZH, Tong JH, Xiao LT, Bai MY, and Xiang FN. 2019b. A GmSIN1/GmNCED3s/GmRbohBs feed-forward loop acts as a signal amplifier that regulates root growth in soybean exposed to salt stress. Plant Cell 31:2107-2130. 10.1105/tpc.18.00662

Liang WJ, Ma XL, Wan P, and Liu LY. 2018. Plant salt-tolerance mechanism: A review. Biochemical and Biophysical Research Communications 495:286-291. 10.1016/j.bbrc.2017.11.043

Liu X, Zhu YM, Zhai H, Cai H, Ji W, Luo X, Li J, and Bai X. 2012. AtPP2CG1, a protein phosphatase 2C, positively regulates salt tolerance of Arabidopsis in abscisic acid-dependent manner. Biochemical and Biophysical Research Communications 422:710-715. 10.1016/j.bbrc.2012.05.064

Liu YJ, Ji XY, Nie XG, Qu M, Zheng L, Tan ZL, Zhao HM, Huo L, Liu SN, Zhang B, and Wang YC. 2015. Arabidopsis AtbHLH112 regulates the expression of genes involved in abiotic stress tolerance by binding to their E-box and GCG-box motifs. New Phytologist 207:692-709. 10.1111/nph.13387

Long L, Yang WW, Liao P, Guo YW, Kumar A, and Gao W. 2019. Transcriptome analysis reveals differentially expressed ERF transcription factors associated with salt response in cotton. Plant Sci 281:72-81. 10.1016/j.plantsci.2019.01.012

Makhloufi E, Yousfi FE, Marande W, Mila I, Hanana M, Berges H, Mzid R, and Bouzayen M. 2014. Isolation and molecular characterization of ERF1, an ethylene response factor gene from durum wheat (Triticum 
turgidum L. subsp. durum), potentially involved in salt-stress responses. J Exp Bot 65:6359-6371. 10.1093/jxb/eru352

Munsif F, Kong XJ, Khan A, Shah TR, Arif M, Jahangir M, Akhtar K, Tang DF, Zheng J, Liao XF, Faisal S, Ali I, Iqbal A, Ahmad P, and Zhou RY. 2020. Identification of differentially expressed genes and pathways in isonuclear kenaf genotypes under salt stress. Physiologia Plantarum. 10.1111/ppl.13253

Nieves-Cordones M, Aleman F, Martinez V, and Rubio F. 2010. The Arabidopsis thaliana HAK5 K ${ }^{+}$ transporter is required for plant growth and $\mathrm{K}^{+}$acquisition from low $\mathrm{K}^{+}$solutions under saline conditions. Molecular Plant 3:326-333. 10.1093/mp/ssp102

Qu Y, Guan R, Bose J, Henderson SW, Wege S, Qiu L, and Gilliham M. 2021. Soybean CHX-type ion transport protein GmSALT3 confers leaf $\mathrm{Na}^{+}$exclusion via a root derived mechanism, and $\mathrm{Cl}^{-}$exclusion via a shoot derived process. Plant Cell Environ 44:856-869. 10.1111/pce.13947

Ren ZH, Gao JP, Li LG, Cai XL, Huang W, Chao DY, Zhu MZ, Wang ZY, Luan S, and Lin HX. 2005. A rice quantitative trait locus for salt tolerance encodes a sodium transporter. Nature Genetics 37:1141-1146. $10.1038 /$ ng 1643

Schachtman DP, and Schroeder JI. 1994. Structure and transport mechanism of a high-affinity potassium uptake transporter from higher-plants. Nature 370:655-658. DOI 10.1038/370655a0

Shi HZ, Quintero FJ, Pardo JM, and Zhu JK. 2002. The putative plasma membrane $\mathrm{Na}^{+} / \mathrm{H}^{+}$antiporter SOS1 controls long-distance $\mathrm{Na}^{+}$transport in plants. Plant Cell 14:465-477. 10.1105/tpc.010371

Shu YJ, Li W, Zhao JY, Liu Y, and Guo CH. 2018. Transcriptome sequencing and expression profiling of genes involved in the response to abiotic stress in Medicago ruthenica. Genetics and Molecular Biology 41:638-648. 10.1590/1678-4685-Gmb-2017-0284

Singh PK, Singh R, and Singh S. 2013. Cinnamic acid induced changes in reactive oxygen species scavenging enzymes and protein profile in maize (Zea mays L.) plants grown under salt stress. Physiology and Molecular Biology of Plants 19:53-59. 10.1007/s12298-012-0126-6

Song QS, Joshi M, and Joshi V. 2020a. Transcriptomic analysis of short-term salt stress response in watermelon seedlings. International Journal of Molecular Sciences 21:6036. 10.3390/ijms21176036

Song Y, Yang W, Fan H, Zhang X, and Sui N. 2020b. TaMYB86B encodes a R2R3-type MYB transcription 
487

factor and enhances salt tolerance in wheat. Plant Sci 300:110624. 10.1016/j.plantsci.2020.110624

Song YS, Li JL, Liu ML, Meng Z, Liu KC, and Sui N. 2019. Nitrogen increases drought tolerance in maize seedlings. Functional Plant Biology 46:350-359. 10.1071/Fp18186

Sun ZX, Wang YN, Mou FP, Tian YP, Chen L, Zhang SL, Jiang Q, and Li X. 2016. Genome-wide small RNA analysis of soybean reveals auxin-responsive microRNAs that are differentially expressed in response to salt stress in root apex. Frontiers in Plant Sci 6:1273. 10.3389/fpls.2015.01273

van Zelm E, Zhang YX, and Testerink C. 2020. Salt tolerance mechanisms of plants. Annual Review of Plant Biology 71:403-433. 10.1146/annurev-arplant-050718-100005

Verbruggen N, and Hermans C. 2008. Proline accumulation in plants: a review. Amino Acids 35:753-759. 10.1007/s00726-008-0061-6

Wang YF, Liao YQ, Wang YQ, Yang JW, Zhang N, and Si HJ. 2020a. Genome-wide identification and expression analysis of StPP2C gene family in response to multiple stresses in potato (Solanum tuberosum L.). Journal of Integrative Agriculture 19:1609-1624. 10.1016/s2095-3119(20)63181-1

Wang Z, Hong YC, Zhu GT, Li YM, Niu QF, Yao JJ, Hua K, Bai JJ, Zhu YF, Shi HZ, Huang SW, and Zhu JK. 2020b. Loss of salt tolerance during tomato domestication conferred by variation in a $\mathrm{Na}^{+} / \mathrm{K}^{+}$transporter. EMBO Journal 39: e103256. 10.15252/embj.2019103256

Wanner LA, Li GQ, Ware D, Somssich IE, and Davis KR. 1995. The phenylalanine ammonia-lyase gene family in Arabidopsis thaliana. Plant Molecular Biology 27:327-338. 10.1007/Bf00020187

Wu B, Hu YN, Huo PJ, Zhang Q, Chen X, and Zhang ZW. 2017. Transcriptome analysis of hexaploid hulless oat in response to salinity stress. PLoS One 12: e0171451. 10.1371/journal.pone.0171451

Yang A, Dai XY, and Zhang WH. 2012. A R2R3-type MYB gene, OsMYB2, is involved in salt, cold, and dehydration tolerance in rice. Journal of Experimental Botany 63:2541-2556. 10.1093/jxb/err431

Yong HY, Zou ZW, Kok EP, Kwan BH, Chow K, Nasu S, Nanzyo M, Kitashiba H, and Nishio T. 2014. Comparative transcriptome analysis of leaves and roots in response to sudden increase in salinity in Brassica napus by RNA-seq. Biomed Research International 2014:467395. 10.1155/2014/467395

Yu ZP, Duan XB, Luo L, Dai SJ, Ding ZJ, and Xia GM. 2020. How plant hormones mediate salt stress responses. Trends in Plant Sci 25:1117-1130. 10.1016/j.tplants.2020.06.008 
514

515

516

517

518

519

520

521

522

523

524

525

526

527

528

529

530

531

532

533

534

535

536

537

538

539

540

Zeng AL, Chen PY, Korth KL, Ping JQ, Thomas J, Wu CJ, Srivastava S, Pereira A, Hancock F, Brye K, and Ma JX. 2019. RNA sequencing analysis of salt tolerance in soybean (Glycine max). Genomics 111:629635. 10.1016/j.ygeno.2018.03.020

Zhang B, Liu J, Yang ZE, Chen EY, Zhang CJ, Zhang XY, and Li FG. 2018. Genome-wide analysis of GRAS transcription factor gene family in Gossypium hirsutum L. BMC Genomics 19:348. 10.1186/s12864-0184722-x

Zhang J, Wang J, Jiang W, Liu J, Yang S, Gai J, and Li Y. 2016. Identification and analysis of $\mathrm{NaHCO}_{3}$ stress responsive genes in wild soybean (Glycine soja) roots by RNA-seq. Front Plant Sci 7:1842. $10.3389 /$ fpls.2016.01842

Zhang WX, Wang N, Yang JT, Guo H, Liu ZH, Zheng XJ, Li S, and Xiang FN. 2020a. The salt-induced transcription factor GmMYB84 confers salinity tolerance in soybean. Plant Sci 291: 110326. 10.1016/j.plantsci.2019.110326

Zhang H, Zhao XB, Sun QX, Yan CX, Wang J, Yuan CL, Li CJ, Shan SH, and Liu FZ. 2020b. Comparative transcriptome analysis reveals molecular defensive mechanism of Arachis hypogaea in response to salt stress. International Journal of Genomics 2020:6524093. 10.1155/2020/6524093

Zhang X, Chen L, Shi Q, and Ren Z. 2020c. SlMYB102, an R2R3-type MYB gene, confers salt tolerance in transgenic tomato. Plant Sci 291:110356. 10.1016/j.plantsci.2019.110356

Zhang P, Wang RL, Yang XP, Ju Q, Li WQ, Lu SY, Tran LSP, and Xu J. 2020d. The R2R3-MYB transcription factor AtMYB49 modulates salt tolerance in Arabidopsis by modulating the cuticle formation and antioxidant defence. Plant Cell and Environment 43:1925-1943. 10.1111/pce.13784

Zhao LJ, Cui JJ, Cai YY, Yang SN, Liu JG, Wang W, Gai JY, Hu ZB, and Li Y. 2020. Comparative transcriptome analysis of two contrasting soybean varieties in response to aluminum toxicity. International Journal of Molecular Sciences 21:4316. 10.3390/ijms21124316

Zhao MJ, Yin LJ, Liu Y, Ma J, Zheng JC, Lan JH, Fu JD, Chen M, Xu ZS, and Ma YZ. 2019a. The ABAinduced soybean ERF transcription factor gene GmERF75 plays a role in enhancing osmotic stress tolerance in Arabidopsis and soybean. BMC Plant Biol 19:506. 10.1186/s12870-019-2066-6

Zhao YY, Yang ZE, Ding YP, Liu LS, Han X, Zhan JJ, Wei X, Diao YY, Qin WQ, Wang P, Liu PP, Sajjad M, 
541 Zhang XL, and Ge XY. 2019b. Over-expression of an R2R3 MYB gene, GhMYB73, increases tolerance $542 \quad$ to salt stress in transgenic Arabidopsis. Plant Sci 286:28-36. 10.1016/j.plantsci.2019.05.021

543 Zhou Y, Yang P, Cui FL, Zhang FT, Luo XD, and Xie JK. 2016. Transcriptome analysis of salt stress 544 responsiveness in the seedlings of Dongxiang wild rice (Oryza rufipogon Griff.). PLoS One 11: e0146242. $545 \quad$ 10.1371/journal.pone.0146242 
Table $\mathbf{1}$ (on next page)

Original RNA-seq data and quality control analysis 
1

2 Table 1 Original RNA-seq data and quality control analysis

\begin{tabular}{llllll}
\hline Sample & Raw reads $(M)$ & Clean reads $(M)$ & Q30 $(\%)$ & Total reads & Total mapped reads \\
\hline CK-1 & 50.49 & 49.3 & 93.26 & 49300196 & $48248935(97.87 \%)$ \\
CK-2 & 50.05 & 48.85 & 93.52 & 48851760 & $47818910(97.89 \%)$ \\
CK-3 & 58.13 & 56.88 & 92.85 & 56884250 & $55613843(97.77 \%)$ \\
NaCl-1 & 52.01 & 50.88 & 93.04 & 50878700 & $49752466(97.79 \%)$ \\
NaCl-2 & 50.2 & 49.11 & 93.02 & 49105610 & $48029838(97.81 \%)$ \\
NaCl-3 & 56.17 & 54.94 & 92.89 & 54943854 & $53713686(97.76 \%)$ \\
Total & 317.05 & 309.96 & & & \\
\hline
\end{tabular}

3 


\section{Table 2 (on next page)}

Classification statistics for DEGs of up-regulated (UR) and down-regulated genes (DR) in in salt-stressed soybeans according to KEGG pathway analysis 
1 Table 2 Classification statistics for DEGs of up-regulated (UR) and down-regulated genes (DR) in in salt2 stressed soybean according to KEGG pathway analysis

\begin{tabular}{lrr}
\hline KEGG categories & Number of UR & Number of DR \\
\hline Cellular Processes & & \\
$\quad$ Transport and catabolism & 1 & \\
Environmental Information Processing & 34 & 29 \\
$\quad$ Signal transduction & 1 & 0 \\
Membrane transport & & \\
Genetic Information Processing & 1 & 14 \\
Translation & 5 & 9 \\
Folding, sorting and degradation & 0 & 5 \\
Transcription & 0 & 3 \\
Replication and repair & & 11 \\
Metabolism & 3 & 8 \\
Nucleotide metabolism & 7 & 4 \\
Metabolism of terpenoids and Polyketides & 2 & 19 \\
Metabolism of other amino acids & 2 & 3 \\
Metabolism of cofactors and vitamins & 10 & 20 \\
Lipid metabolism & 4 & 13 \\
Energy metabolism & 28 & 16 \\
Carbohydrate metabolism & & \\
Biosynthesis of other secondary & 12 & \\
Metabolites & 16 & \\
Amino acid metabolism & & \\
Organismal Systems & 4 & \\
Environmental adaptation & & \\
\hline
\end{tabular}

3 


\section{Figure 1}

Effects of salt stress on growth and physiological indicators of soybean seedlings.

(A) After salt treatment $(0,3,6,12 \mathrm{~h})$, the accumulation of $\mathrm{O}_{2}{ }^{-}$and $\mathrm{H}_{2} \mathrm{O}_{2}$ in leaves was detected by NBT and DAB staining, respectively, (B) phenotypic changes of soybean seedlings after treatment with $100 \mathrm{mM} \mathrm{NaCl}$ for 6 days, (C) plant height, (D) fresh weight, (E) $\mathrm{Na}^{+}$content and $(\mathrm{F}) \mathrm{K}^{+}$content in shoots, and $(\mathrm{G})$ proline content under normal $(\mathrm{CK})$ and salt treatment conditions. Data represent mean $\pm S D(n=6), * P<0.05$ (Student's $t$ test).

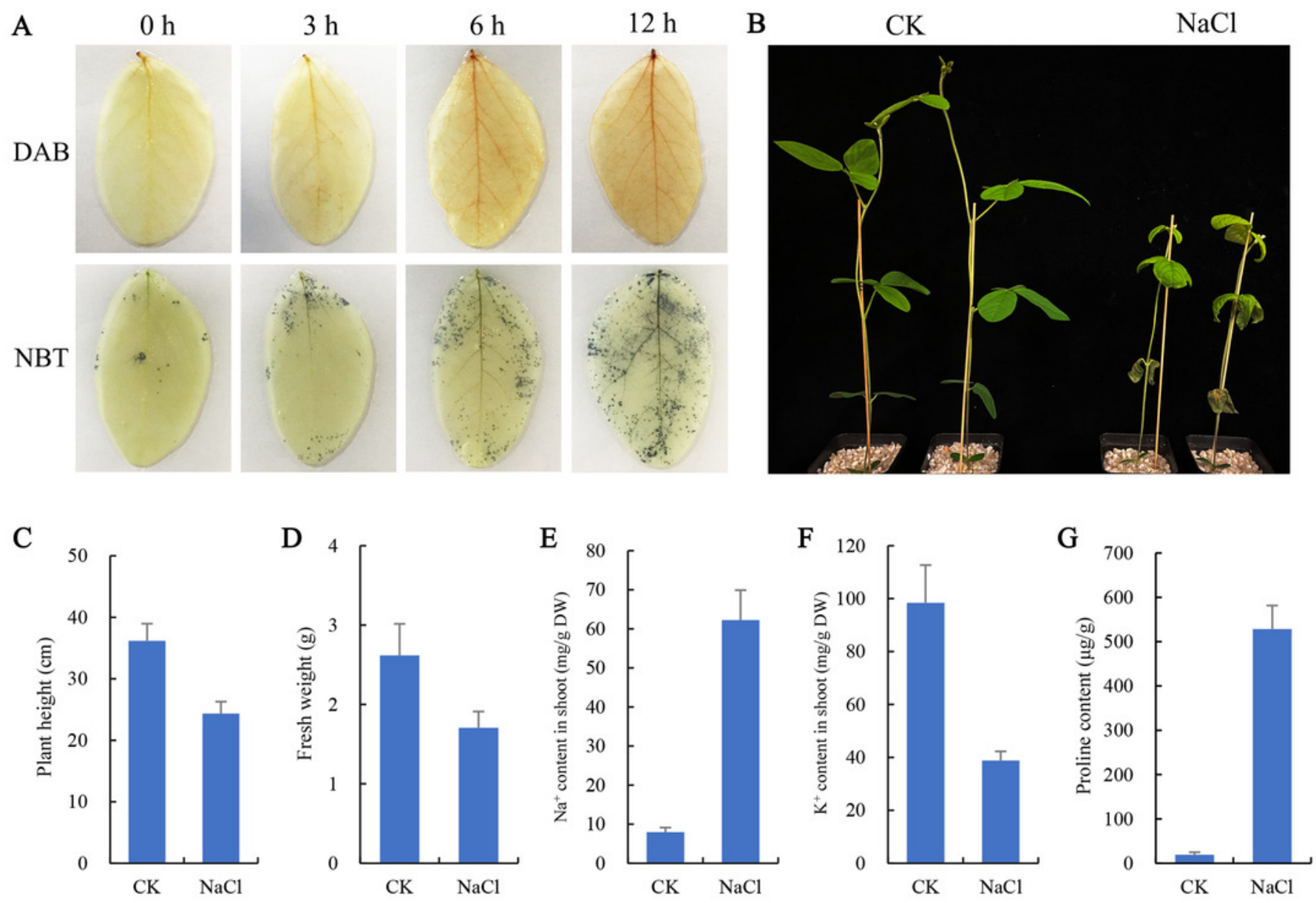


Figure 2

RNA-seq analysis of soybean shoots under salt stress.

(A) Correlation analysis of control (CK) and salt-treated samples ( $\mathrm{NaCl}),(B)$ the number of genes with different expression levels in different samples, and (C) volcano plot of DEGs. Red dots indicate up-regulated genes and blue dots indicate down-regulated genes. (D) Correlations in qRT-PCR (x-axis) RNA-seq data (y-axis) using 10 randomly selected DEGs.
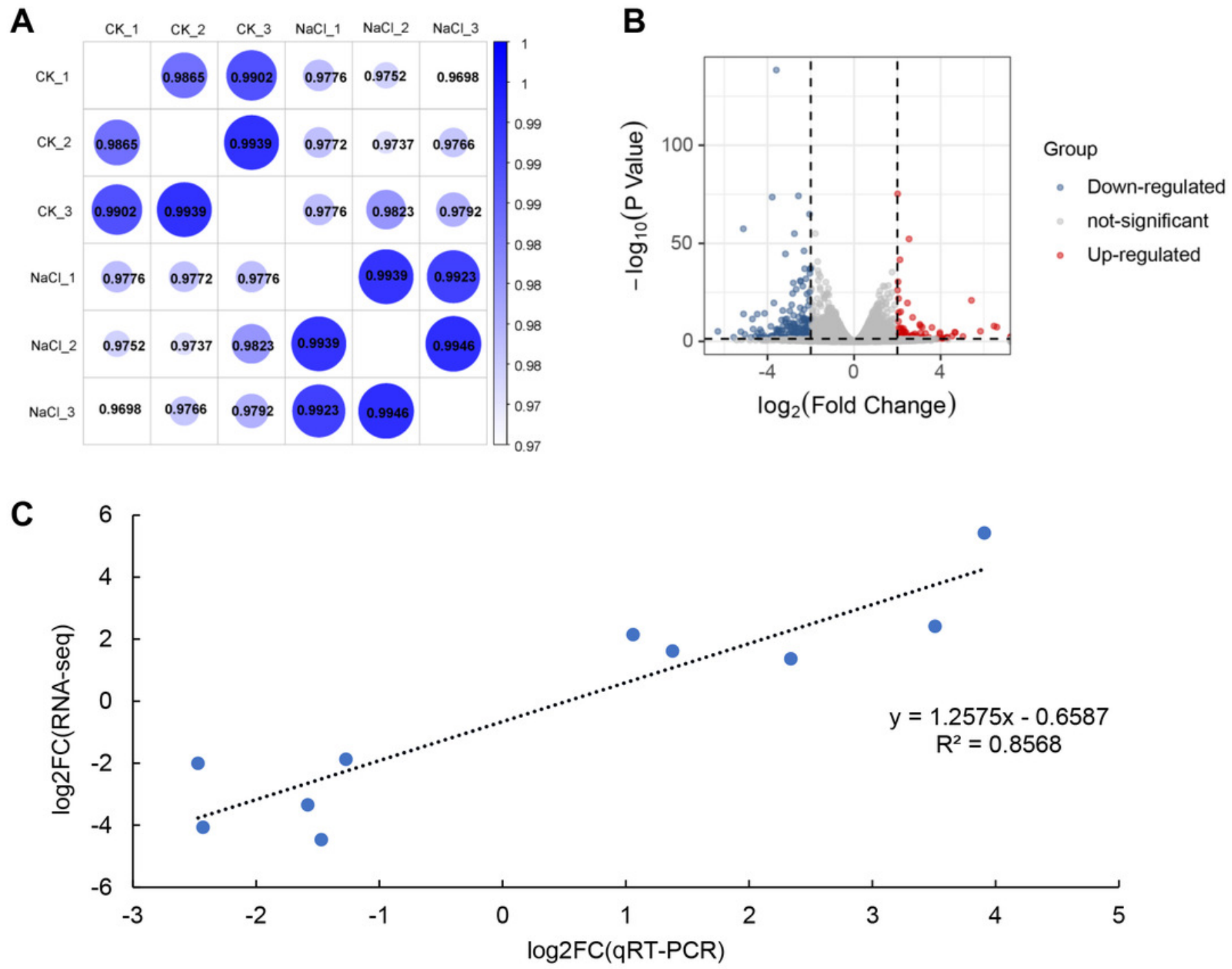
Figure 3

Kyoto Encyclopedia of Genes and Genomes (KEGG) analysis of DEGs in response to salt stress using the top twenty significant enrichment KEGG terms.

(A) KEGG terms enriched by up-regulated DEGs, (B) KEGG terms enriched by down-regulated DEGs. 
A

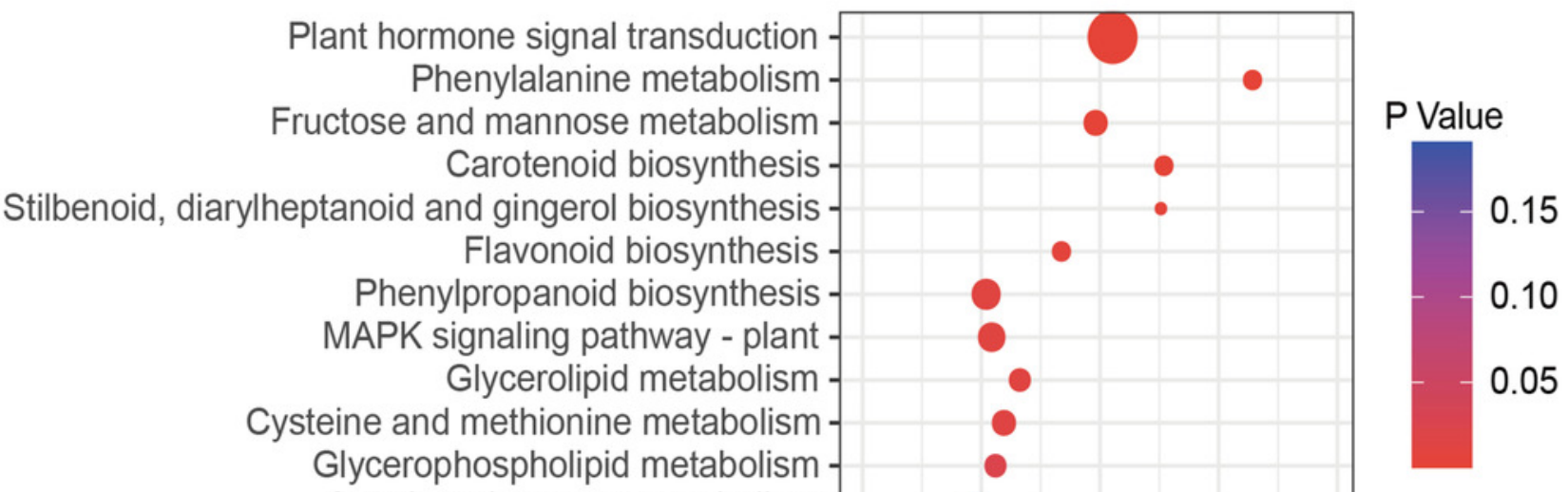

$\stackrel{\frac{E}{1}}{ \pm}$

Starch and sucrose metabolism

Circadian rhythm - plant

Galactose metabolism

Pentose phosphate pathway

Carbon fixation in photosynthetic organisms Inositol phosphate metabolism

Glycolysis / Gluconeogenesis

Purine metabolism

Amino sugar and nucleotide sugar metabolism

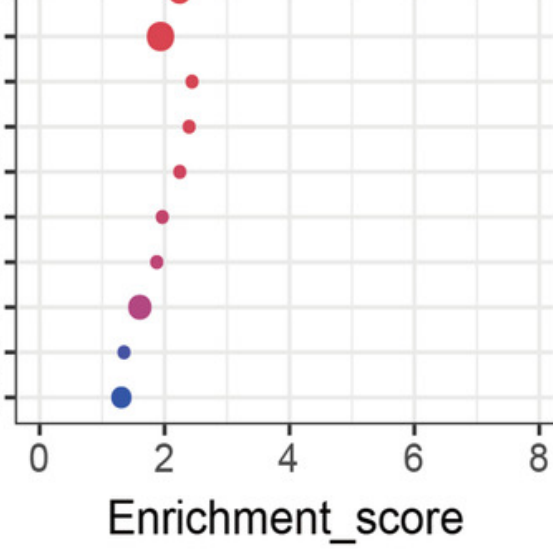

Number

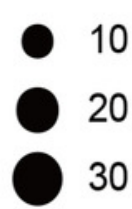

B

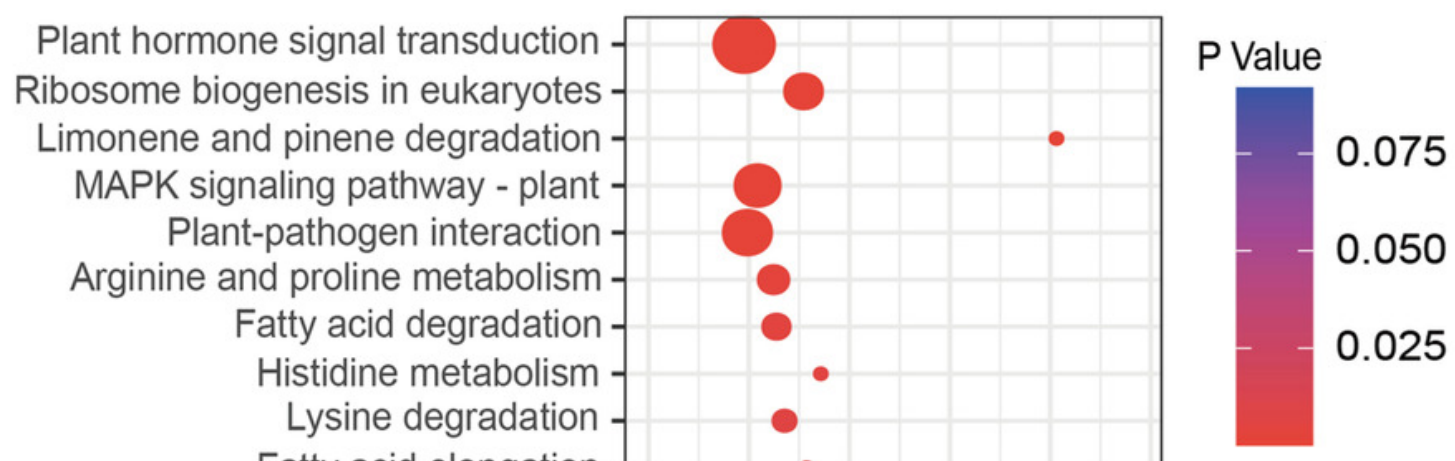

$\underset{\frac{L}{\Phi}}{\Phi}$

Diterpenoid biosynthesis

Glycerolipid metabolism

Ascorbate and aldarate metabolism Valine, leucine and isoleucine degradation

Glycolysis / Gluconeogenesis

Phenylpropanoid biosynthesis Pentose and glucuronate interconversions

Tryptophan metabolism

Beta-Alanine metabolism

Peroxisome

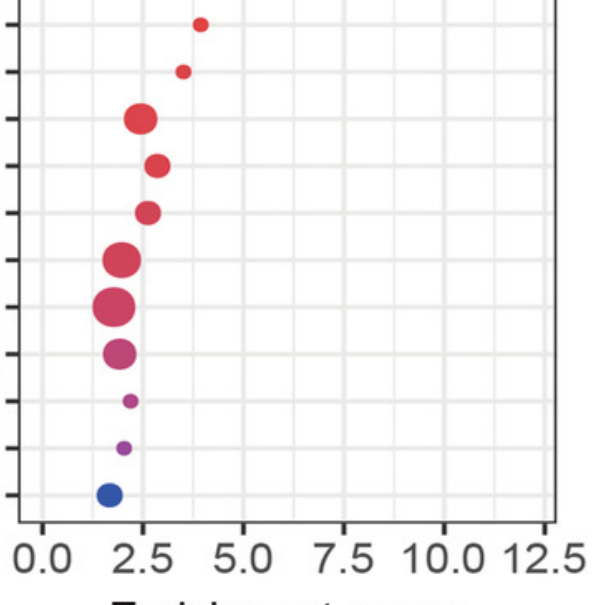

Enrichment_score
Number

- 5

10

15

20

25 


\section{Figure 4}

Key KEGG pathways of related genes in response to salt stress and salt-induced expression patterns.

(A) Plant hormone signal transduction, (B) MAPK signaling pathway, (C) MAPK signaling pathway, (D) phenylalanine metabolism, and (E-H) PYR/PYLs, PP2Cs, proline dehydrogenase genes, and phenylalanine ammonia-lyase gene expression represented using the FPKM value. 


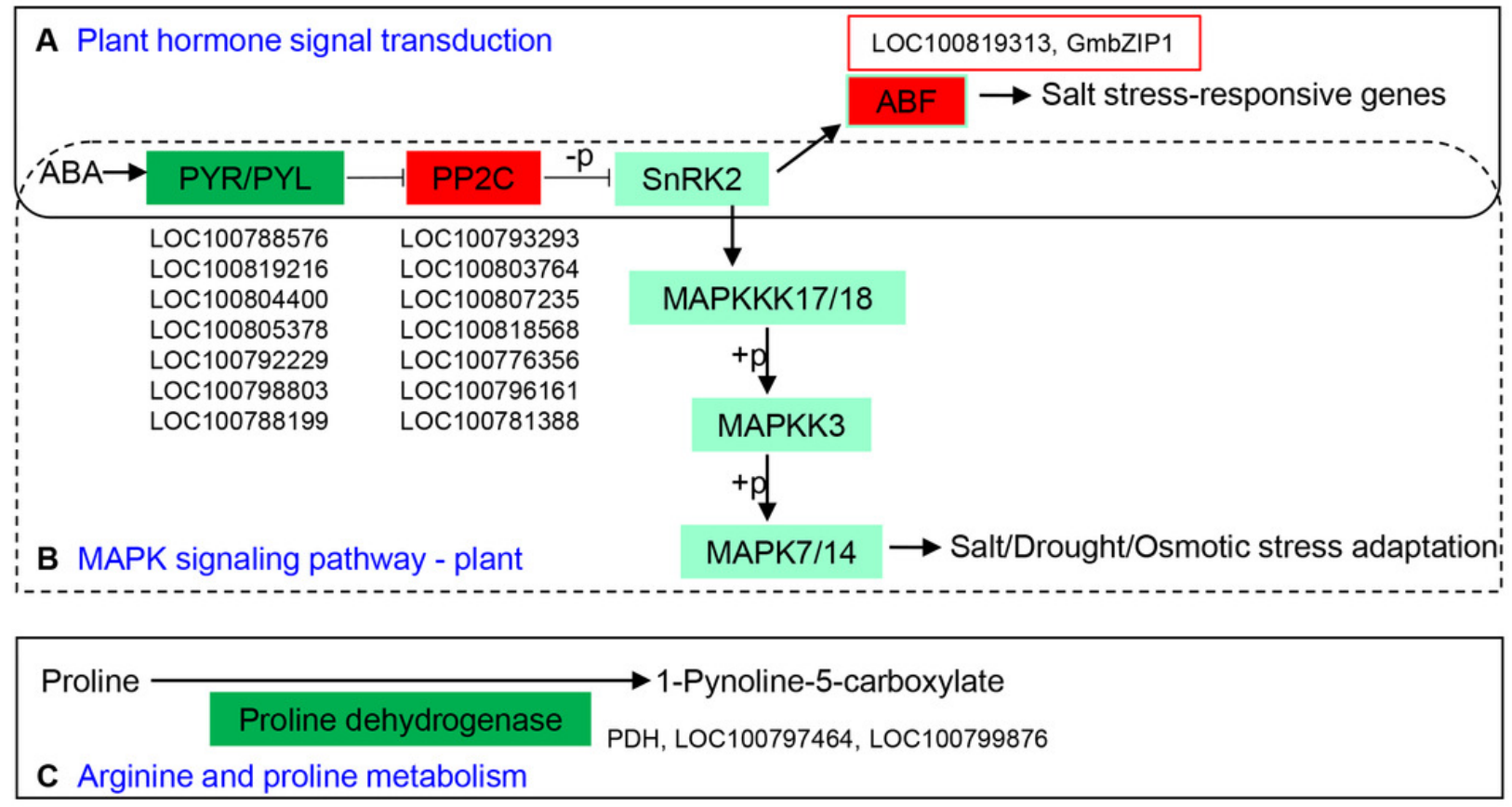

Phenylalanine $\longrightarrow$ phenylalanine ammonia-lyase
D PmPAL1.2, GmPAL1.3, GmPAL2.2, GmPAL2.4
D Phenylalanine metabolism
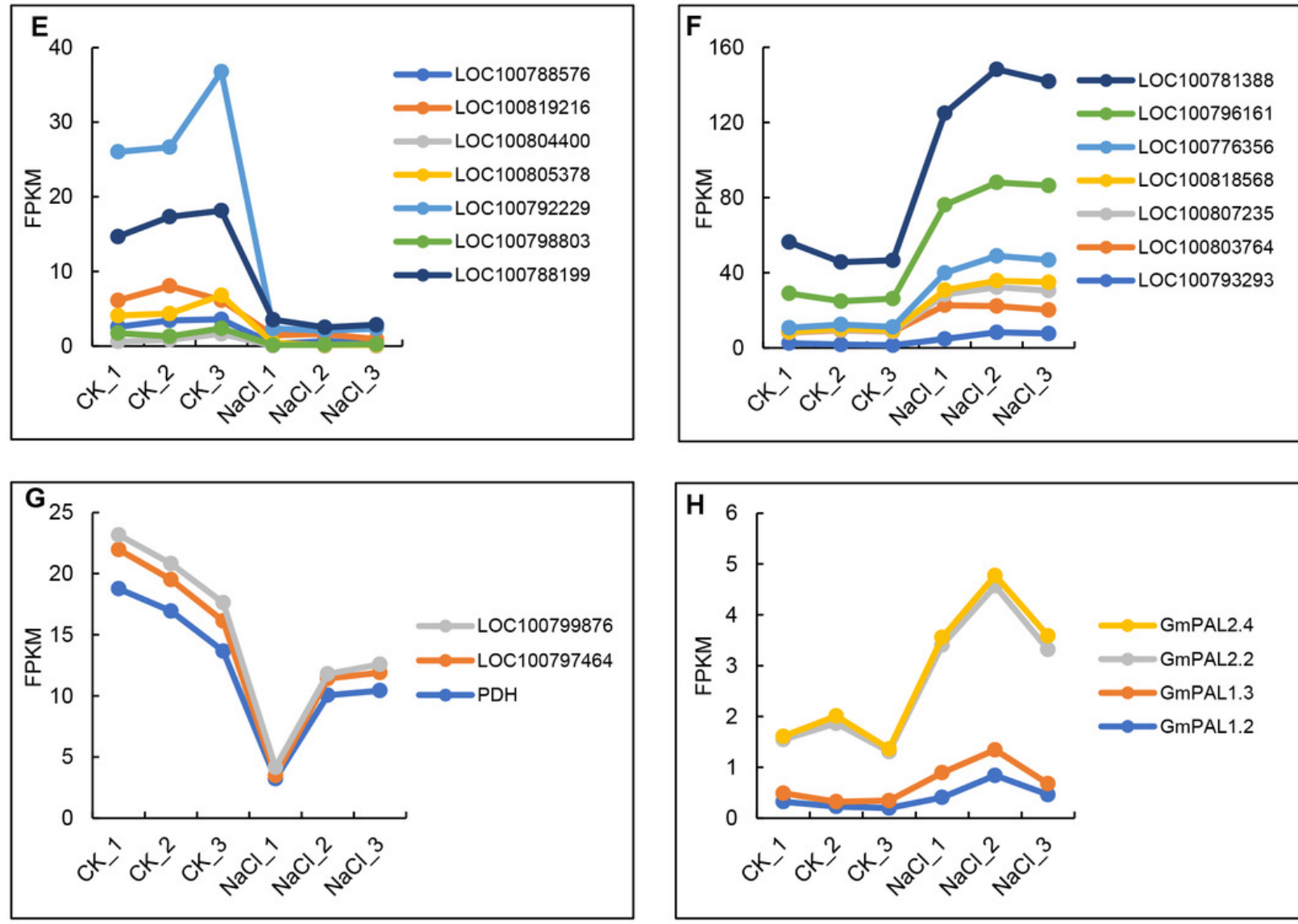


\section{Figure 5}

Differentially expressed TFs induced by salt stress in soybean.

(A) The classification and number of differentially expressed TFs, (B) classification of upregulated and down-regulated TFs.

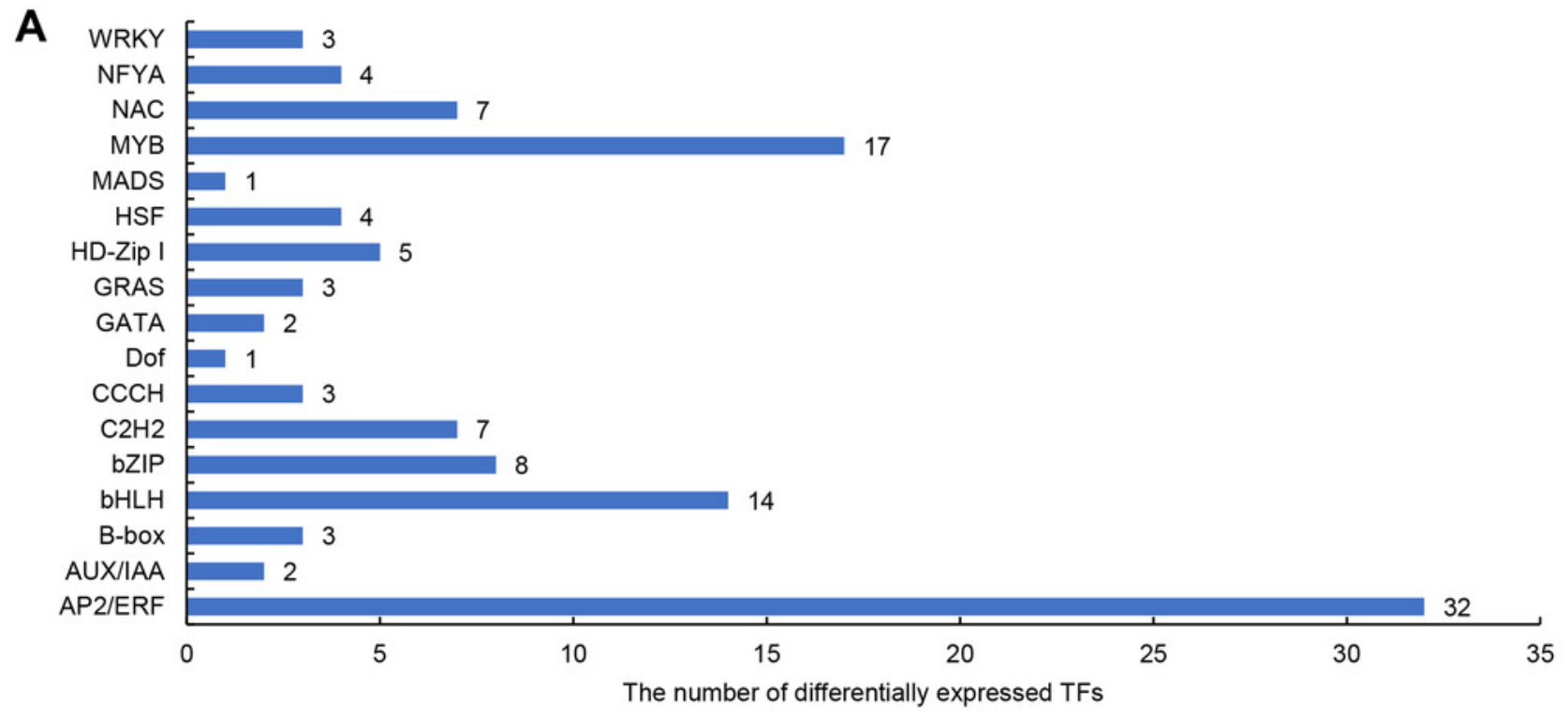

B
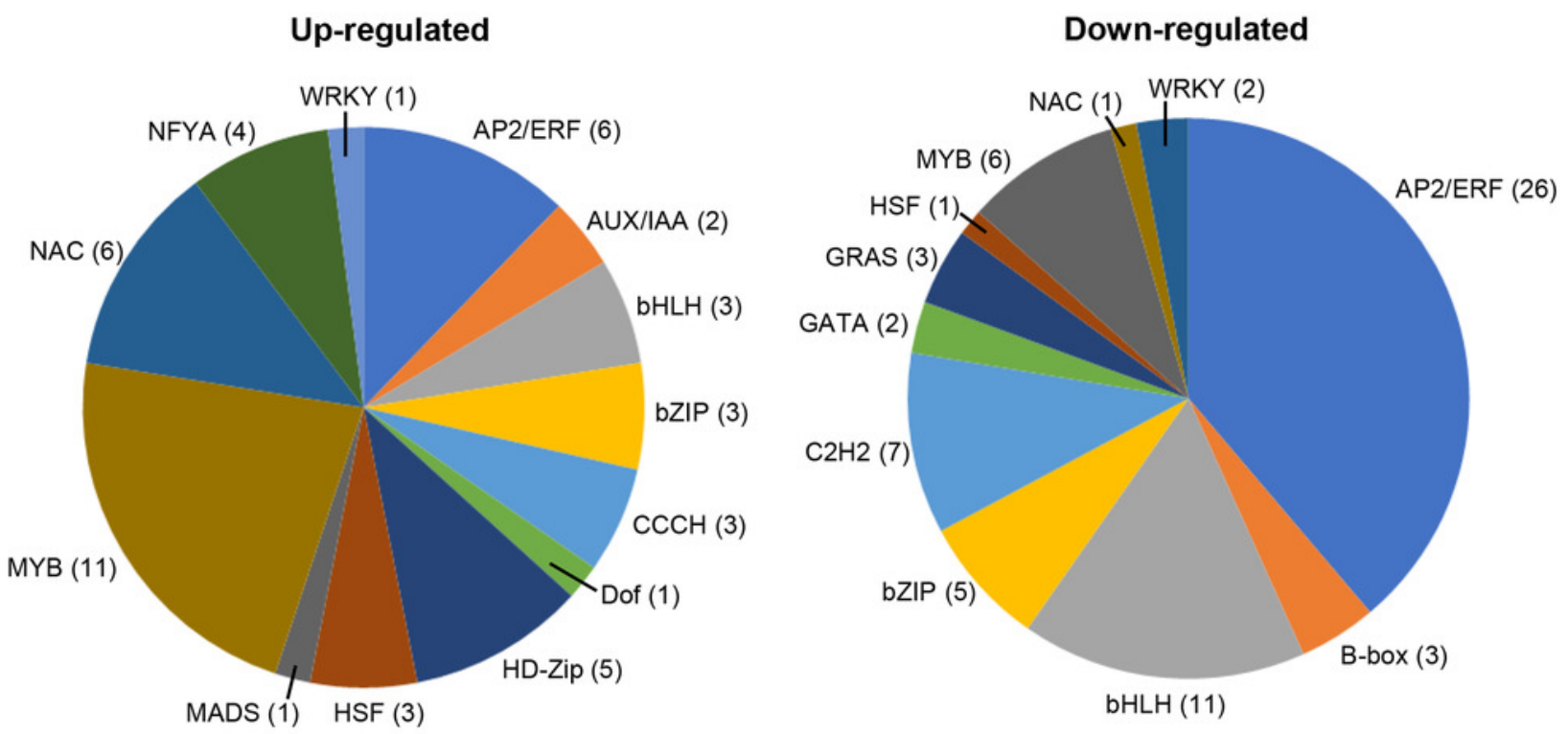


\title{
Figure 6
}

\begin{abstract}
(A) The classification and number of differentially expressed TFs, (B) classification of upregulated and down-regulated TFs.
\end{abstract}

(A) The phylogenetic tree of differentially expressed MYBs and other reported MYBs involved in abiotic stress, (B) heatmap diagrams showing the relative expression levels of differentially expressed MYBs based on the FPKM values, $(C)$ expression analysis of GmMYB46 induced by $\mathrm{NaCl}$ and mannitol, (D) subcellular localization of GmMYB46 in tobacco leaves. 


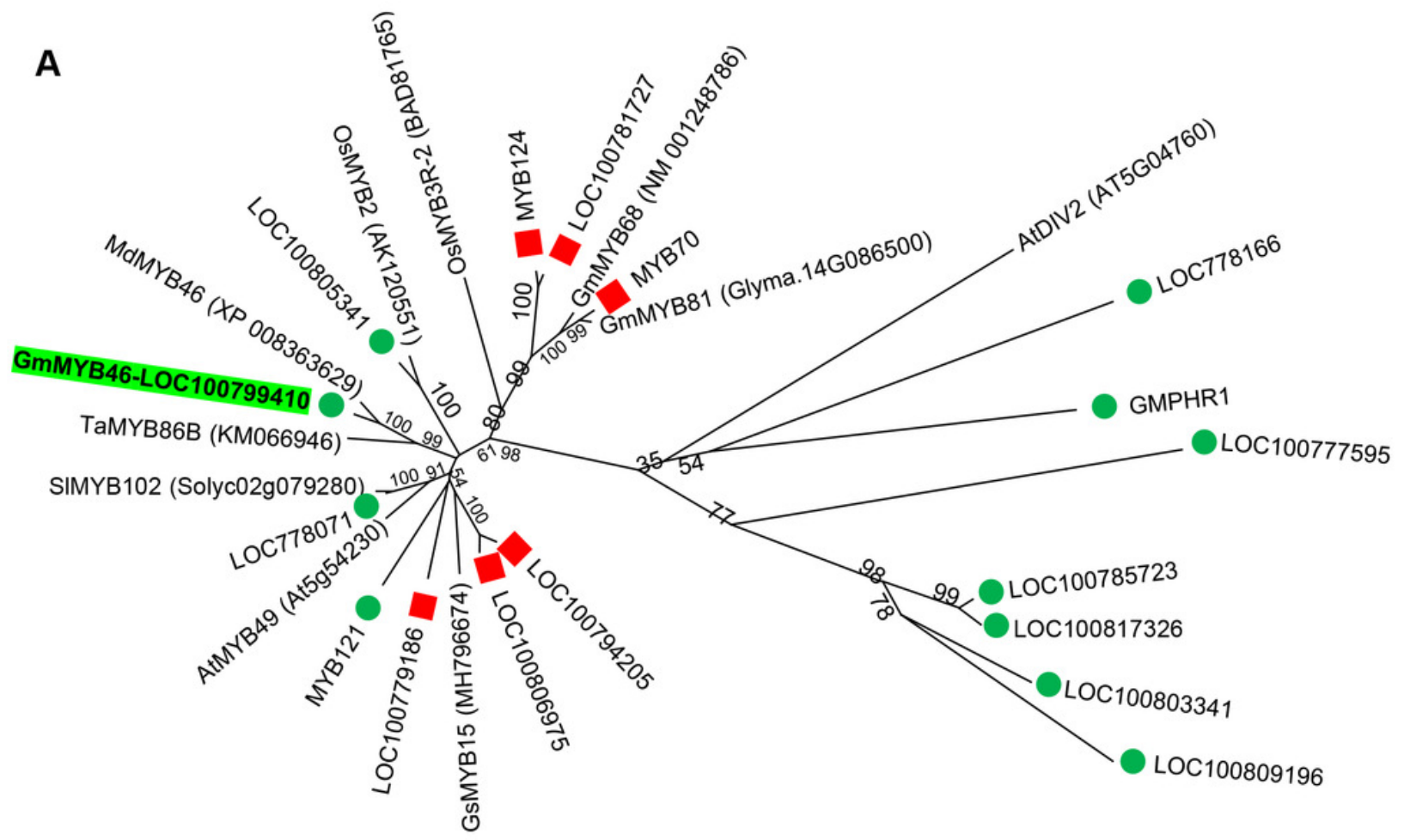

B

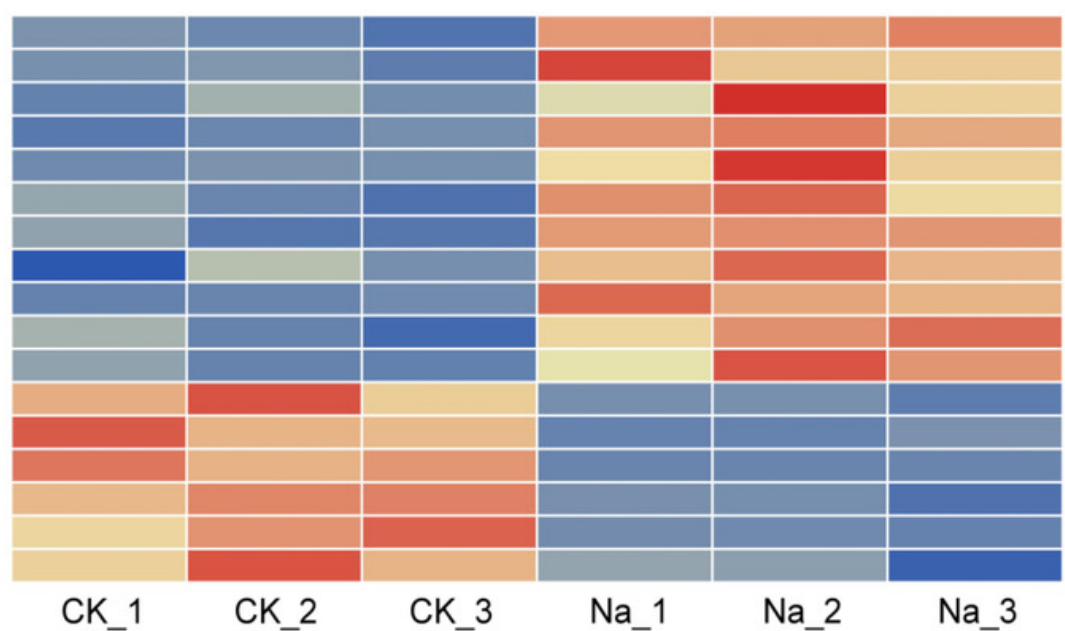

Gene ID

(NCBI database) Fold Change

LOC778071 2.07

MYB121 2.31

LOC100805341 4.09

LOC100799410

LOC100809196

LOC100803341

GMPHR1

LOC100777595

LOC778166

LOC100785723

LOC100817326

LOC100794205

LOC100806975

LOC100779186

MYB124

MYB70

LOC100781727

CK_1 CK_2 $\quad$ CK_3 $\quad \mathrm{Na}$-1 $\quad \mathrm{Na}$ 2 $\quad \mathrm{Na}$ 3

\section{C}

$\mathrm{NaCl} \mathrm{Oh}$

$3 \mathrm{~h}$

$6 \mathrm{~h}$

$12 \mathrm{~h}$

GmEF-1a

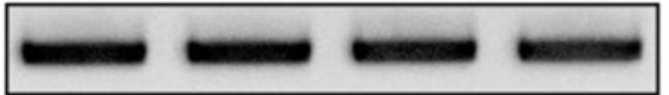

GmMYB46

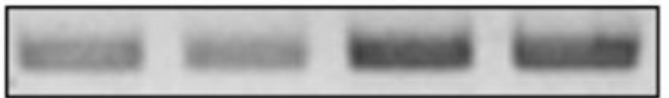

Mannitol $\mathrm{Oh}$

$3 \mathrm{~h}$

$6 \mathrm{~h}$

$12 \mathrm{~h}$

GmEF-1a

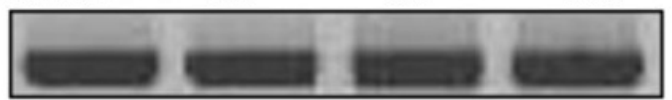

GmMYB46

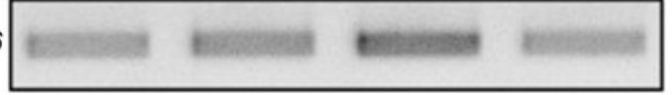

D

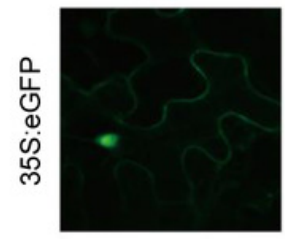

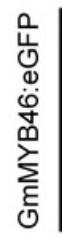

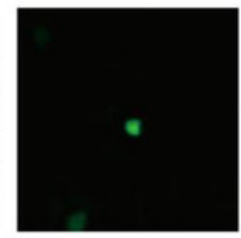

Bright
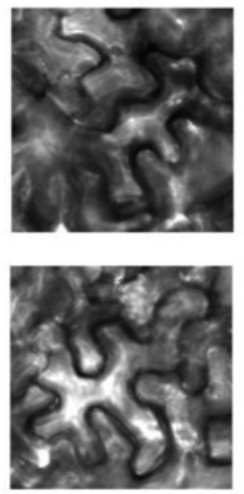

7.49
2.25

2.23

2.44

2.06

3.15

2.01

2.99

0.14

0.03

0.00

0.30

0.43

0.25

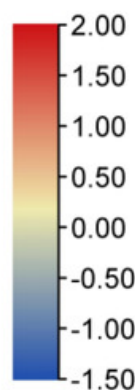




\section{Figure 7}

Overexpression of GmMYB46 enhanced the salt tolerance of transgenic Arabidopsis.

(A) Phenotypic analysis of wide-type (Col-0) and overexpressed lines (OX46.1 and OX46.2),

(B) survival rate of Arabidopsis seedlings, (C) relative water content, (D) MDA content, (E)

proline content, and (F) enzyme activities of SOD and POD. The data represent the mean \pm SD. $* \mathrm{P}<0.05$ (Student's t test). 

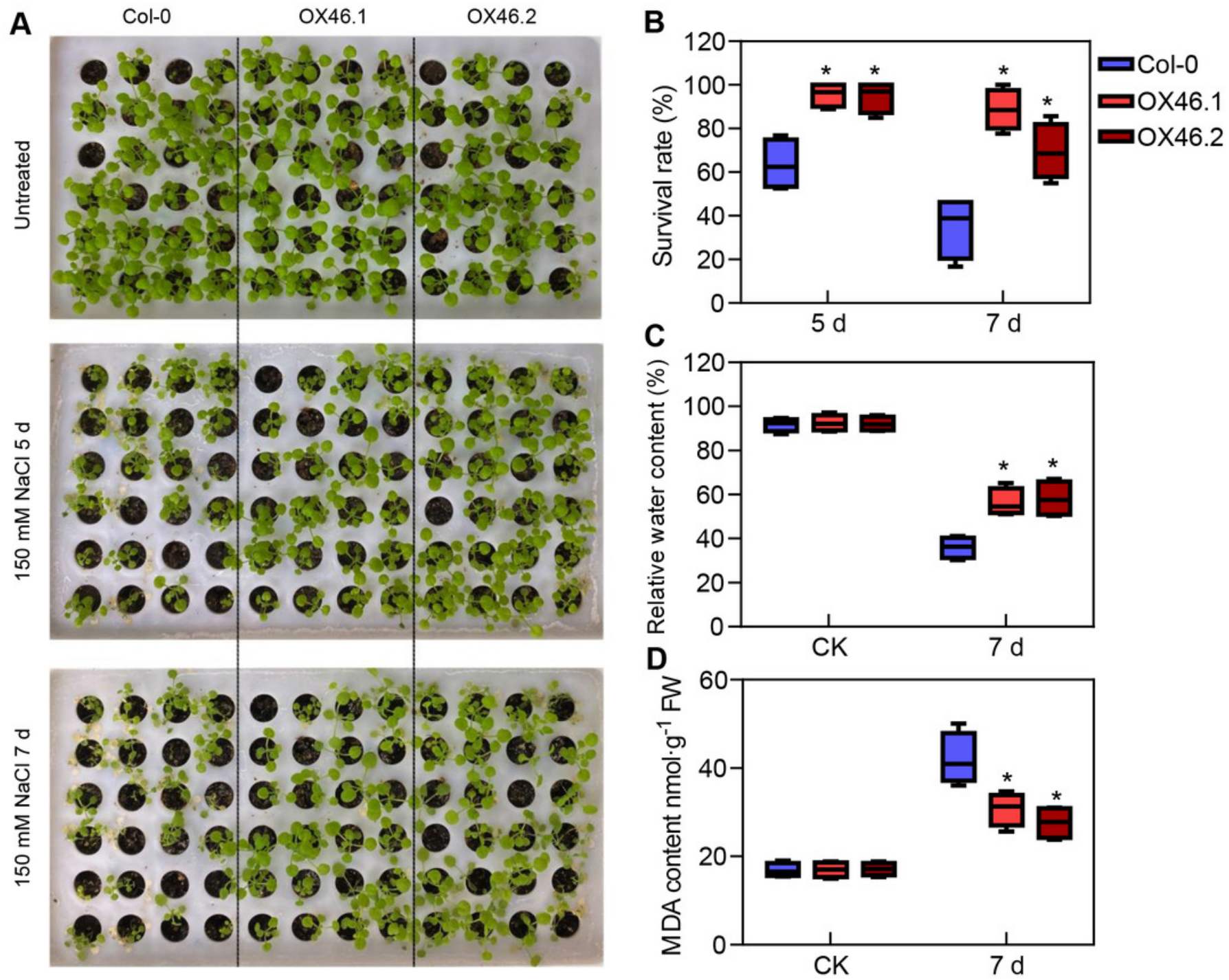

E

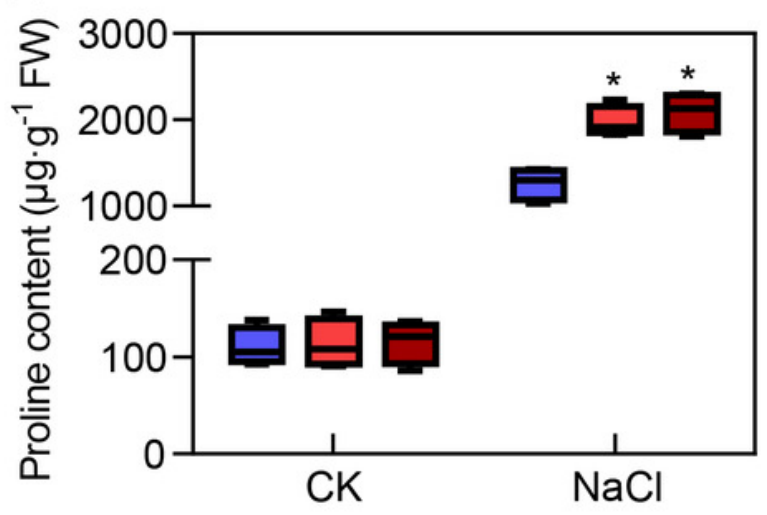

$\mathbf{F}$

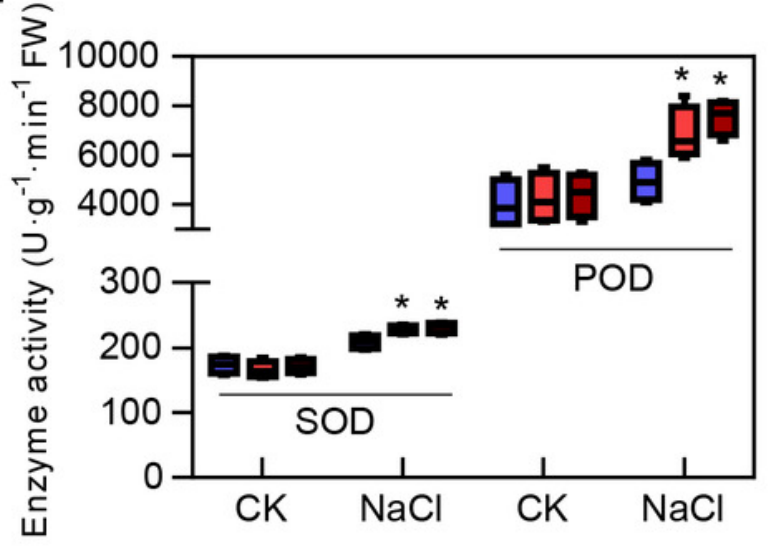


Figure 8

Expression patterns of stress-responsive genes in Col-0 and overexpressed lines (OX46.1 and OX46.2) in response to salt stress.

The expression of (A) P5CS1, (B) SOD, (C) POD, and NCED3 genes.
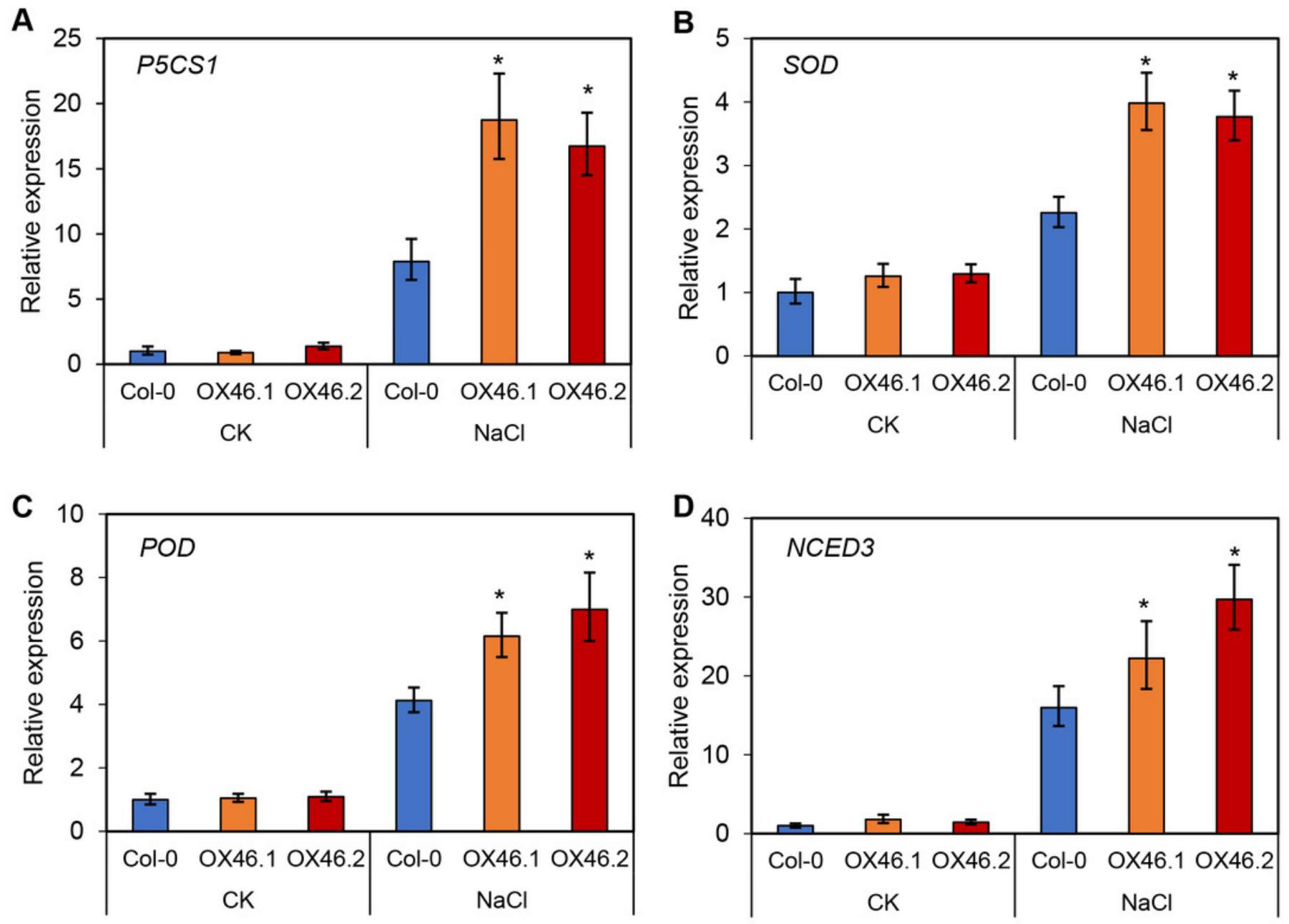